\title{
Metal Bis(Acetylide) Complex Molecular Wires: Concepts and Design Strategies
}

David C. Milan, ${ }^{\mathrm{a}}$ Andrea Vezzoli, ${ }^{\mathrm{a}}$ Inco J. Planje, ${ }^{\mathrm{a}}$ Paul J. Low ${ }^{*}{ }^{\mathrm{b}}$

a. Department of Chemistry, University of Liverpool, Crown St, Liverpool, L69 7ZD, UK

b. School of Molecular Sciences, University of Western Australia, 35 Stirling Highway, Crawley, 6009,WA, Australia.Email: paul.low@uwa.edu.au; Tel: +61 864883045

\begin{abstract}
The past decade has seen a remarkable surge in studies of thin-film and singlemolecule electronics, due in no small part to the development and advacement of experimental methods for the construction and measurement of metal|molecule|metal junctions. Within the plethora of molecular structures that have been investigated, metal complexes of general form trans-M $(\mathrm{C} \equiv \mathrm{CR})_{2}\left(\mathrm{~L}_{\mathrm{n}}\right)$ have attracted attention from the inorganic and organometallic chemistry community in the search for efficient molecular wires due to the potential $\pi-d-\pi$ orbital mixing along the molecular backbone. In this article progress towards this goal will be summarised, and design strategies for future molecular components discussed.
\end{abstract}

\section{Introduction}

The idea of using molecules to perform useful electronic functions within device structures has attracted attention for decades, with the first expression of the concept perhaps being attributable to von Hippel, popularised by Feynman, and brought to the attention of the wider chemical community by Aviram and Ratner. ${ }^{1,2}$ The development of methods through which to contact thin films or single molecules to macroscopic (usually metal) electrodes and directly measure through-molecule conductance in the resulting metal|molecule|metal molecular junctions (Figure 1) has given further impetus to the design and development of molecular components. Consequently, molecules that display electronic behaviour analogous to that of conventional wires, switches, rectifiers and transistors within molecular junctions have now been developed., 
4 In addition, these studies have opened new avenues for exploration and exploitation of molecular electronic characteristics, including thermoelectric properties, ${ }^{5,6}$ chemically and redox gated charge transport ${ }^{7-9}$ and spintronics, ${ }^{10-}$ ${ }^{13}$ as well as offering insight into the fundamentals of charge transfer processes within and through molecules. ${ }^{14,15}$

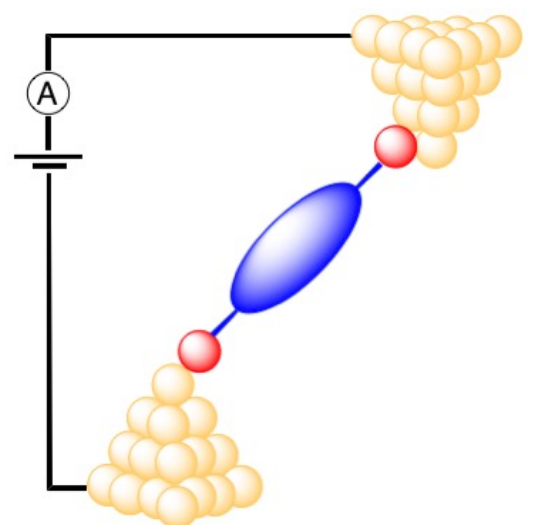

Figure 1. A schematic representation of a single molecule junction. The pyramids represent the electrodes, the blue object the molecular backbone and the red spheres the functional groups contacting to the electrode surfaces.

Before describing the electronic response of molecules within a junction in more detail, it is useful to consider some simple descriptions of charge transport in molecular junctions and the associated electrical characteristics. The conductance, $G$, through a molecular junction can be described in terms of a simplified form of the Landauer-Büttiker transmission model where

$$
G=\frac{2 e^{2}}{h} \frac{4 \Gamma^{2}}{\left(E_{F}-E_{M O}\right)^{2}+4 \Gamma^{2}}
$$

From the model, several key points can be highlighted. Firstly, conductance, $G$, which is proportional to the transmission function, $T(\mathrm{E})$, through the junction therefore depends on both the energy offset between a critical frontier molecular orbital and the electrode Fermi level $\left(\left|\mathrm{E}_{\mathrm{F}}-\mathrm{E}_{\mathrm{MO}}\right|\right)$; a better match between the orbital energy and the Fermi level leads to higher conductance 
(Figure 2). Secondly, strong molecule-electrode coupling $(\Gamma)$ leads to a broadening of the molecular orbitals through hybridisation with the electrode. As the molecular levels broaden, the integrated area under Equation 1 within the bias window increases, and thus the conductance increases. Finally, we note that anchor groups that effectively couple with the electrodes and increase $\Gamma$ lead to broader transmission resonances, and higher conductance. It is therefore unsurprising that conductance across a molecule within a molecular junction is a complex function of the chemical composition of the molecular backbone, the anchor group, fine details of the molecule-surface contact and the precise molecular geometry within the junction. ${ }^{16,17}$

(a)

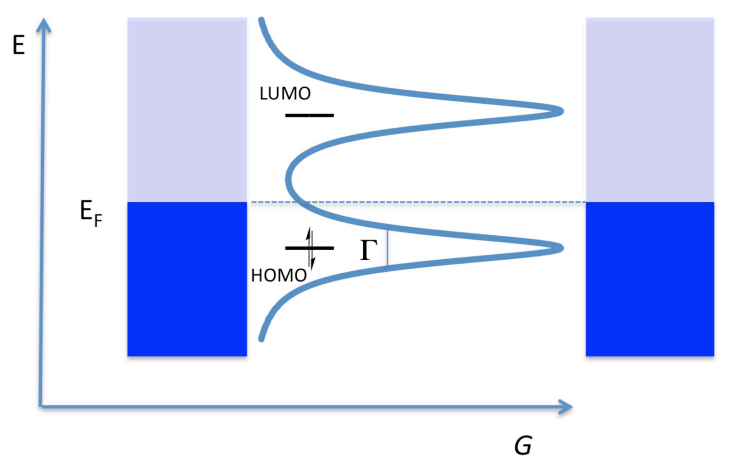

(b)

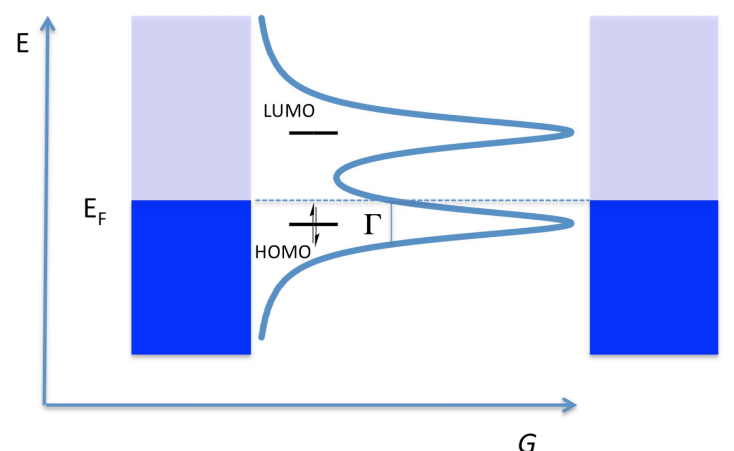

(c)

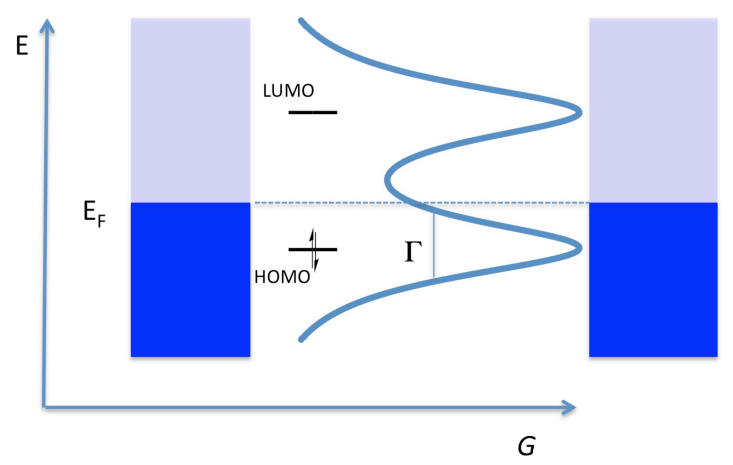


Figure 2. (a) A plot of conductance (G) from the zero-bias Landauer-Büttiker model, as a function of the energy of the HOMO and LUMO, taken as two representative molecular orbitals. At the Fermi level, conductance (G) increases (b) as the HOMO energy rises closer to the Fermi level, and / or (c) as coupling $(\Gamma)$ increases.

Molecular conductance, $G$, is often expressed in relation to the quantum of conductance, $G_{0}$, where

$$
G_{0}=2 e^{2} / h=77,480 n S
$$

( $e=$ electron charge, $h=$ Planck's constant), which is the conductance of a single gold-atom contact. ${ }^{18-20}$ Given that molecular conductance is highly dependent on both the nature of the molecule-surface contact ${ }^{21-23}$ and the solvent environment, ${ }^{24,25}$ it is not at all uncommon for multiple conductance values to be determined for any given molecule, which can make comparisons of conductivity between different molecular species difficult. Nevertheless, some broad generalisations serve to highlight the range of molecular conductance values that have been accessed. Thus, conductance values for single molecules as high as $1-10^{-1} G_{0}$ have been reported for aromatic molecules contacted through the $\pi$-face, ${ }^{26-28}$ carbon-contacted butanediyl $\left(\mathrm{Au}\left|\mathrm{CH}_{2} \mathrm{CH}_{2} \mathrm{CH}_{2} \mathrm{CH}_{2}\right| \mathrm{Au}\right),{ }^{29}$ and xylyl $\left(\mathrm{Au}\left|\mathrm{CH}_{2} \mathrm{C}_{6} \mathrm{H}_{4} \mathrm{CH}_{2}\right| \mathrm{Au}\right)$ junctions ${ }^{30}$ and $\mathrm{C}_{60} \cdot{ }^{31,32}$ Values of up to $10^{-2}-10^{-3} G_{0}$ are found for thiolate-contacted shortchain alkanes, ${ }^{33-35}$ oligoynes, ${ }^{36}$ tolanes, ${ }^{37}$ thioether- ${ }^{38}$ or anilinide-contacted ${ }^{39}$ biaryls, and cyclic thioether-contacted biphenylenes. ${ }^{40}$ Conductance values of $10^{-4}-10^{-5}$ are common for families of longer wires based on well-explored molecular backbones such as 1,4-bis(phenylethynyl)benzenes, ${ }^{41-44}$ and oligoynes of comparable length. ${ }^{36,45}$

In seeking to compare and ultimately optimise the wire-like performance of molecules within molecular junctions, it is also useful to consider conductance as a function of molecular length. For tunnelling based charge transport mechanisms, along a homologous series, $G$ decays exponentially with distance, 
$l$, such that $G \propto e^{-\beta l} G_{0} \cdot{ }^{46}$ The decay constant, $\beta$, which has units of reciprocal length, is correlated with the molecular HOMO-LUMO gap, ${ }^{46}$ and varies from values as high as $12.3 \mathrm{~nm}^{-1}$ for siloxanes, $7.5 \mathrm{~nm}^{-1}$ for alkanes and $3.9 \mathrm{~nm}^{-1}$ for silanes, ${ }^{47}$ to $3-1 \mathrm{~nm}^{-1}$ for rigid conjugated organic compounds such as oligo(phenylene ethynylenes) (OPEs), ${ }^{42,43,48}$ oligo(phenylene imines), ${ }^{49}$ and oligo(thiophenes). ${ }^{50}$ Even lower $\beta$ values have been reported for butadiynelinked oligo(porphyrin)s $\left(0.4 \mathrm{~nm}^{-1}\right)^{51,52}$ and oligoynes $\left(0.3-0.1 \mathrm{~nm}^{-1}\right) .^{25,36,45}$

\section{Metal bis(acetylide) complexes as components for molecular electronics}

With these various features in mind, considerable attention has been turned to the electrical behaviour of metal complexes within molecular junctions. ${ }^{53-55}$ This article sets out to summarise the potential of organometallic transbis(acetylide) complexes, trans $-\mathrm{M}(\mathrm{C} \equiv \mathrm{CR})_{2}\left(\mathrm{~L}_{\mathrm{n}}\right)$, which offer a range of useful physical and chemical features not readily accessed in purely organic systems, ${ }^{56}$ as components in molecular electronics. For example, the metal $d_{\mathrm{xz}}$ and $d_{\mathrm{yz}}$ orbitals offer the correct $\pi$-type symmetry to interact with the acetylide $\pi$ orbitals, giving an extended, $\pi$-conjugated electronic structure (Figure 3 ). The energy and occupancy of these $d$-orbitals, the extent of $d$ - $\pi$ mixing and the molecular geometry can be tuned through judicious choice of metal, $\mathrm{M}$. Electronic properties such as the energy of the HOMOs and LUMOs, and related properties including redox potentials and the HOMO-LUMO gap, can be fine-tuned by the choice of metal, molecular contacts and ancillary ligands, L. 


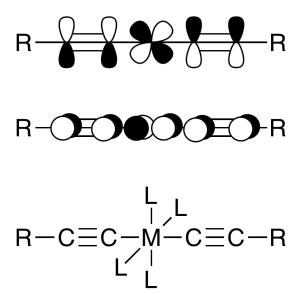

Figure 3. A schematic of the frontier orbitals of a generic octahedral $d^{6}$ trans$M(C \equiv C R)_{2} L_{4}$ complex illustrating the $\pi-d-\pi$ overlaps in the HOMO and HOMO-1

A common justification for the use of metal complexes within molecular junctions is that the relatively high-lying HOMO will be closer to the electrode Fermi level, and result in higher conductance than an otherwise analogous organic compound. In addition, physical properties of the metal complexes such as solubility and molecular volume, the latter being important in the context of the available geometries within a single-molecule junction or the packing efficiency within the monolayer films that comprise 'large-area' junctions, can also be tuned through the ancillary ligands. The acetylide ligands, $\mathrm{C} \equiv \mathrm{CR}$, can be readily extended through a variety of cross-coupling strategies, to create longer structures, and functionalised to carry a wide-range of surface-contacting groups. Together these features give an exceptional versatile design platform, and there is now a sufficiently large body of experimental information available to allow these ideas and the efficacy of the various design strategies to be assessed and revised.

\section{Setting the scene: molecular conductance studies of ruthenium bis(acetylide) complexes}

Ruthenium complexes trans $-\mathrm{Ru}(\mathrm{C} \equiv \mathrm{CR})_{2}(\mathrm{PP})_{2}(\mathrm{PP}=$ bis(phosphine) ligand such as bis(dimethylphosphino)ethane (dmpe), bis(diphenylphosphino)methane (dppm), bis(diphenylphosphino)ethane (dppe) etc) offer a relatively high lying HOMO delocalised over the long molecular axis with considerable $\pi-d-\pi$ character, and low HOMO-LUMO gaps making them attractive moieties for use in the construction of molecular wires. ${ }^{57}$ As indicated above, the anchor 
group and the molecule-electrode coupling also play a critical role in the electrical response of a molecular junction. The thiolate surface contact has been widely used in molecular junction studies, due the strength of the Au-S bond and the strong coupling / hybridisation that takes place. ${ }^{16}$ Thiolate is also known to promote HOMO-mediated conduction channels,${ }^{58}$ making it an excellent choice for use as an anchor group with metal complex-based compounds that are designed to promote HOMO-mediated conductance.

However, free thiols, R-SH, have a strong tendency to oxidatively homo-couple to dithiolates, R-S-S-R. Consequently, the thiol contacting group is usually introduced via the thioacetate (R-SAc) or ethyltrimethylsilyl (R$\mathrm{SCH}_{2} \mathrm{CH}_{2} \mathrm{SiMe}_{3}$ ) protected derivatives. Thiolates also form a wide range of surface contacts on gold, ${ }^{59-61}$ often resulting in determination of multiple conductance values. Sulfur-gold bonds also rupture and interconvert stochastically, leading to characteristic 'blinking' effects in scanning probe images of thiolate-contacted molecules within inert supporting monolayer. Thus, whilst thiolate remains a valuable anchor group in molecular electronics research, the limitations have naturally led to interest in systems bearing alternative surface contacting groups that might simplify the conductance profile of the system under investigation, tune the energy level alignment and control the molecule-electrode-coupling. 

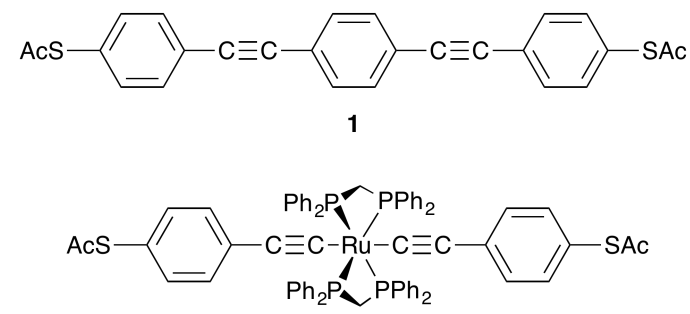

2

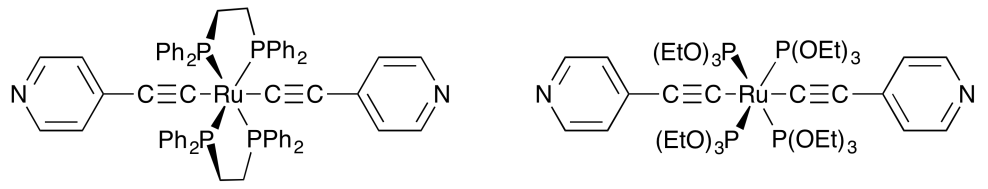

$3 a$

3b

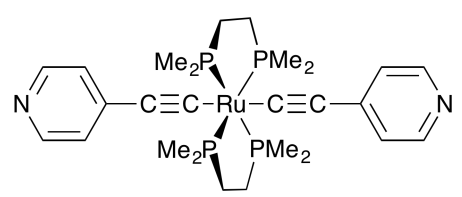

3c

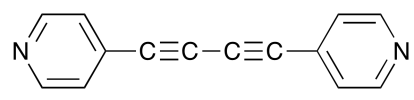

4

Figure 4. The compounds and complexes 1 - 4

Following removal of the protecting acetyl group in situ, single-molecule STM break-junction (STM-BJ) studies of the reference compound 1,4-(4$\left.\mathrm{AcSC}_{6} \mathrm{H}_{4} \mathrm{C} \equiv \mathrm{C}\right)_{2} \mathrm{C}_{6} \mathrm{H}_{4}$ (1) and the octahedral $\mathrm{d}^{6}$ ruthenium complexes trans$\mathrm{Ru}\left(\mathrm{C} \equiv \mathrm{CC}_{6} \mathrm{H}_{4} \mathrm{SAc}\right)_{2}(\mathrm{dppm})_{2}(2)$ (Figure 4) at low bias $(-50$ or $-100 \mathrm{mV})$ have given molecular conductances of $(0.46 \pm 0.26) \times 10^{-4} \mathrm{G}_{0}$ and $(2.4 \pm 0.9) \times 10^{-4}$ $\mathrm{G}_{0}$, respectively (Table 1). ${ }^{62}$ The conductance of the ruthenium complex 2 is thus measurably greater than that of the organic compound $\mathbf{1}$, even allowing for the shorter molecular length and realistic $\beta$ values. In seeking to explain the relative conductance values, an analysis of $\ln \left(I / V^{2}\right)$ vs $\ln (1 / V)$ was undertaken, revealing a lower transition voltage (corresponding to a lower tunnel barrier height or offset between the molecular and Fermi levels) for $2(0.25 \pm 0.03 \mathrm{eV})$ than the organic model compound $1(0.66 \pm 0.11 \mathrm{eV})$. Assessment of $\beta$ using an STM apparent height based method also confirmed lower relative values for the ruthenium complex $2\left(1.01 \pm 0.25 \AA^{-1}\right)$ than $1\left(1.11 \pm 018 \AA^{-1}\right)$. However, these values of the decay constant are likely rather influenced by the method of 
assessment and the approximations used in the calculation, and will not compare well with values determined from, for example, plots of conductance vs length in a systematically elongated homologous series.

The 4-pyridylethynyl complexes trans $-\mathrm{Ru}(\mathrm{C} \equiv \mathrm{Cpy})_{2}\left(\mathrm{~L}_{\mathrm{n}}\right)\left(\mathrm{L}_{\mathrm{n}}=(\mathrm{dppe})_{2} \quad(\mathbf{3 a})\right.$, $\left.\left(\mathrm{P}(\mathrm{OMe})_{3}\right)_{4}(\mathbf{3 b}),(\mathrm{dmpe})_{2}(\mathbf{3 c})\right)$ have been prepared and studied using STM-BJ in tetraglyme solution, and conductance values may be compared with the 1,4bis(4-pyridyl)-buta-1,3-diyne (4) studied under identical conditions (Figure 4). ${ }^{63}$ The metal complexes $\mathbf{3 a}$ - $\mathbf{c}$ gave rise to conductance values that are largely insensitive to the nature of the ancillary ligands $\left(3 \mathrm{a}:(2.5 \pm 0.04) \times 10^{-4}\right.$ $\left.G_{0} ; 3 \mathbf{b}(3.8 \pm 0.08) \times 10^{-4} G_{0} ; 3 \mathbf{c}(3.9 \pm 0.3) \times 10^{-4} G_{0}\right)$, and some $2-4$ fold greater than the diyne model $4\left((1.0 \pm 0.2) \times 10^{-4} G_{0}\right)$, despite the shorter length of the diyne. DFT calculations carried out using relatively small gold pyramids $\left(\mathrm{Au}_{35}\right)$ as models for the electrodes were consistent with HOMO-mediated tunnelling as the dominant conductance mechanism, despite the usual behaviour of pyridyl contacted (organic) molecules to display LUMO-mediated conductance. However, the trends in the conductance values did not follow simple relationships with the HOMO energy or HOMO-LUMO gap, ${ }^{63}$ highlighting the fact that multiple, inter-related parameters influence throughmolecule conductance in a molecular junction.

Although great care must be taken in comparing molecular conductance values collected using different measurement methods and under different conditions due to the impact of various factors including but not limited to the molecular geometry within the junction, electrode surface roughness and composition, and solvent environment, it is interesting to note that the conductance of pyridylcontacted 3a - c is essentially identical to that determined a decade earlier for the thiolate contacted analogue trans $-\mathrm{Ru}\left(\mathrm{C} \equiv \mathrm{CC}_{6} \mathrm{H}_{4} \mathrm{SAc}\right)_{2}(\mathrm{dppm})_{2}$ (2). The similarly composed wire-like ruthenium complex trans$\mathrm{Ru}\left(\mathrm{C} \equiv \mathrm{CC}_{6} \mathrm{H}_{4} \mathrm{C} \equiv \mathrm{CSiMe}_{3}\right)_{2}(\mathrm{dppe})_{2}$ (5) bearing the trimethylsilylethynyl surface contacting group, which also normally promotes transmission through LUMOtype channels in organic compounds, gives a conductance value of $(5.10 \pm$ 0.99) $\times 10^{-5} G_{0}$ in $\operatorname{STM}-I(s)$ measurements, some 2-fold greater than the 
comparable OPE 1,4-(4- $\left.\mathrm{Me}_{3} \mathrm{SiC} \equiv \mathrm{CC}_{6} \mathrm{H}_{4} \mathrm{C} \equiv \mathrm{C}\right){ }_{2} \mathrm{C}_{6} \mathrm{H}_{4}\left(6:(2.75 \pm 0.56) \times 10^{-5} G_{0}\right)$ (Figure 5). ${ }^{64}$

The greater molecular length of $\mathbf{5}$ and $\mathbf{6}$ readily accounts for the lower conductance values observed for these compounds when compared with $\mathbf{1}, \mathbf{2}$ and 3. However, given the similar molecular lengths of 5 (Si...Si $23.97 \AA$ ) and 6 (Si...Si $24.49 \AA$ ) and identical contacting groups, the increased conductance through the ruthenium complex 5 relative to the organic OPE 6 seems to be an inherent effect due to the difference in the molecular backbone. From these studies it follows that the introduction of the 'electron-rich' $d^{6}$ ruthenium fragment in place of a phenylene ring results in a $2-4$ fold increase in conductance.

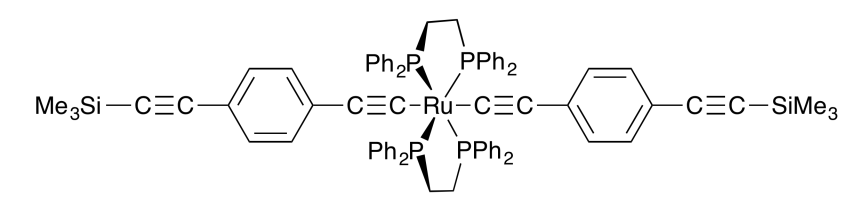

5

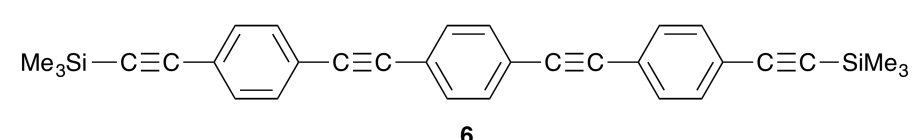

Figure 5 The complex and compound 5 and $\mathbf{6}$ 
Table 1 Summary of single molecule conductance values from trans-bis(acetylide) metal complexes, and methods employed.

\begin{tabular}{|c|c|c|c|c|c|}
\hline & Compound & $G / \mathrm{nS}$ & $G / G_{0}$ & method & ref \\
\hline 1 & $1,4-\left(4-\mathrm{AcSC}_{6} \mathrm{H}_{4} \mathrm{C} \equiv \mathrm{C}\right)_{2} \mathrm{C}_{6} \mathrm{H}_{4}$ & $3.6 \pm 2.0$ & $(0.46 \pm 0.26) \times 10^{-4}$ & STM-BJ & 62 \\
\hline 2 & trans $-\mathrm{Ru}\left(\mathrm{C} \equiv \mathrm{CC}_{6} \mathrm{H}_{4} \mathrm{SAc}\right)_{2}(\mathrm{dppm})_{2}$ & $19 \pm 7$ & $(2.4 \pm 0.9) \times 10^{-4}$ & STM-BJ & 62 \\
\hline $3 \mathbf{a}$ & trans $-\mathrm{Ru}(\mathrm{C} \equiv \mathrm{Cpy})_{2}(\mathrm{dppe})_{2}$ & $19.4 \pm 0.3$ & $(2.5 \pm 0.04) \times 10^{-4}$ & STM-BJ & 63 \\
\hline $\mathbf{3 b}$ & trans $-\mathrm{Ru}(\mathrm{C} \equiv \mathrm{Cpy})_{2}\left\{\mathrm{P}(\mathrm{OMe})_{3}\right\}_{4}$ & $29.4 \pm 0.6$ & $(3.8 \pm 0.08) \times 10^{-4}$ & STM-BJ & 63 \\
\hline $3 c$ & trans $-\mathrm{Ru}(\mathrm{C} \equiv \mathrm{Cpy})_{2}(\mathrm{dmpe})_{2}$ & $30.2 \pm 2.3$ & $(3.9 \pm 0.3) \times 10^{-4}$ & STM-BJ & 63 \\
\hline 4 & $\mathrm{pyC} \equiv \mathrm{CC} \equiv \mathrm{Cpy}$ & $7.7 \pm 1.5$ & $(1.0 \pm 0.2) \times 10^{-4}$ & STM-BJ & 63 \\
\hline 5 & trans $-\mathrm{Ru}\left(\mathrm{C} \equiv \mathrm{CC}_{6} \mathrm{H}_{4} \mathrm{C} \equiv \mathrm{CSiMe}_{3}\right)_{2}(\mathrm{dppe})_{2}$ & $3.95 \pm 0.77$ & $(5.10 \pm 0.99) \times 10^{-5}$ & $\mathrm{STM}-I(s)$ & 64 \\
\hline 6 & $1,4-\left(4-\mathrm{Me}_{3} \mathrm{SiC} \equiv \mathrm{CC}_{6} \mathrm{H}_{4}\right)_{2} \mathrm{C}_{6} \mathrm{H}_{4}$ & $2.13 \pm 0.43$ & $(2.75 \pm 0.56) \times 10^{-5}$ & STM-I $(s)$ & 64 \\
\hline $7 a$ & trans $-\mathrm{Pt}\left(\mathrm{C} \equiv \mathrm{CC}_{6} \mathrm{H}_{4} \mathrm{SAc}\right)_{2}\left(\mathrm{PPh}_{3}\right)_{2}$ & $0.2-0.02$ & $2.6 \times 10^{-6}-2.6 \times 10^{-7}$ & $\mathrm{MCBJ}^{\mathrm{a}}$ & 65 \\
\hline 8 & trans $-\mathrm{Pt}\left(\mathrm{C} \equiv \mathrm{CC}_{6} \mathrm{H}_{4} \mathrm{C} \equiv \mathrm{CSiMe}_{3}\right)_{2}\left(\mathrm{PPh}_{3}\right)_{2}$ & $6.1 \pm 0.85$ & $(7.9 \pm 1.1) \times 10^{-5}$ & $\mathrm{STM}-I(s)^{\mathrm{b}}$ & 66 \\
\hline 9 & trans $-\mathrm{Pt}\left(\mathrm{C} \equiv \mathrm{CC}_{6} \mathrm{H}_{4} \mathrm{C} \equiv \mathrm{CSiMe}_{3}\right)_{2}\left(\mathrm{PEt}_{3}\right)_{2}$ & $2.5 \pm 1.0$ & $(3.2 \pm 1.3) \times 10^{-5}$ & $\mathrm{STM}-I(s)$ & 66 \\
\hline 10 & trans $-\mathrm{Pt}\left\{\mathrm{C} \equiv \mathrm{C}\left(\mathrm{OC}_{6} \mathrm{H}_{13}\right) \mathrm{C} \equiv \mathrm{CSiMe}_{3}\right\}_{2}\left(\mathrm{PPh}_{3}\right)_{2}$ & $2.4 \pm 0.7$ & $(3.1 \pm 0.9) \times 10^{-5}$ & $\mathrm{STM}-I(s)$ & 66 \\
\hline 11 & trans $-\mathrm{Pt}\left\{\mathrm{C} \equiv \mathrm{C}\left(\mathrm{OC}_{6} \mathrm{H}_{13}\right) \mathrm{C} \equiv \mathrm{CSiMe}_{3}\right\}_{2}\left(\mathrm{PEt}_{3}\right)_{2}$ & $2.5 \pm 0.6$ & $(3.2 \pm 0.8) \times 10^{-5}$ & $\mathrm{STM}-I(s)$ & 66 \\
\hline 12 & trans $-\mathrm{Pt}(\mathrm{C} \equiv \mathrm{CC} \equiv \mathrm{CSiMe})_{2}\left(\mathrm{PPh}_{3}\right)_{2}$ & $4.0 \pm 1.2$ & $(5.2 \pm 1.6) \times 10^{-5}$ & $\mathrm{STM}-I(s)^{\mathrm{b}}$ & 66 \\
\hline 13 & trans $-\mathrm{Pt}\left(\mathrm{C} \equiv \mathrm{CC} \equiv \mathrm{CSiMe}_{3}\right)_{2}\left(\mathrm{PEt}_{3}\right)_{2}$ & $3.8 \pm 1.2$ & $(4.9 \pm 1.6) \times 10^{-5}$ & $\mathrm{STM}-I(s)$ & 66 \\
\hline 14 & $\mathrm{Me}_{3} \mathrm{Si}(\mathrm{C} \equiv \mathrm{C})_{4} \mathrm{SiMe}_{3}$ & 1.1 & $1.42 \times 10^{-5}$ & $\mathrm{STM}-I(s)$ & 25 \\
\hline 15 & $\mathrm{Me}_{3} \mathrm{Si}(\mathrm{C} \equiv \mathrm{C})_{5} \mathrm{SiMe}_{3}$ & 0.7 & $0.90 \times 10^{-5}$ & $\mathrm{STM}-I(s)$ & 25 \\
\hline 16 & $\mathrm{py}(\mathrm{C} \equiv \mathrm{C})_{4} \mathrm{py}$ & $3.1 \pm 0.6$ & $(3.98 \pm 0.8) \times 10^{-5}$ & STM-BJ & 36 \\
\hline 17 & $\mathrm{NCC}_{6} \mathrm{H}_{4}(\mathrm{C} \equiv \mathrm{C})_{4} \mathrm{C}_{6} \mathrm{H}_{4} \mathrm{CN}$ & $0.3 \pm 0.1$ & $(0.4 \pm 0.1) \times 10^{-5}$ & STM-BJ & 36 \\
\hline 18 & $\mathrm{BT}(\mathrm{C} \equiv \mathrm{C})_{4} \mathrm{BT}$ & $15.4 \pm 4.0$ & $(2.0 \pm 0.5) \times 10^{-4}$ & STM-BJ & 36 \\
\hline \multirow[t]{2}{*}{19} & $1,4-\left(3-\mathrm{SC}_{4} \mathrm{H}_{3} \mathrm{C} \equiv \mathrm{C}\right)_{2} \mathrm{C}_{6} \mathrm{H}_{4}$ & $3.25 \pm 0.77$ & $(0.42 \pm 0.10) \times 10^{-4}$ & STM-I $(s)$ & 67 \\
\hline & & $21.9 \pm 5.0$ & $(2.83 \pm 0.65) \times 10^{-4}$ & STM-BJ & 67 \\
\hline \multirow[t]{2}{*}{20} & trans $-\mathrm{Pt}\left(\mathrm{C} \equiv \mathrm{CC}_{4} \mathrm{H}_{3} \mathrm{~S}\right)_{2}\left(\mathrm{PEt}_{3}\right)_{2}$ & $5.96 \pm 1.08$ & $(0.77 \pm 0.14) \times 10^{-4}$ & STM-I $(s)$ & 67 \\
\hline & & $20.1 \pm 5.1$ & $(2.70 \pm 0.66) \times 10^{-4}$ & STM-BJ & 67 \\
\hline \multirow[t]{2}{*}{21} & trans $-\mathrm{Ru}\left(\mathrm{C} \equiv \mathrm{CC}_{4} \mathrm{H}_{3} \mathrm{~S}\right)_{2}$ (dppe $)_{2}$ & $7.98 \pm 2.17$ & $(1.03 \pm 0.28) \times 10^{-4}$ & STM-I $(s)$ & 67 \\
\hline & & $24.6 \pm 8.0$ & $(3.18 \pm 1.04) \times 10^{-4}$ & STM-BJ & 67 \\
\hline \multirow[t]{2}{*}{22} & trans $-\mathrm{Ru}\left(\mathrm{C} \equiv \mathrm{CC}_{4} \mathrm{H}_{3} \mathrm{~S}\right)_{2}\left\{\mathrm{P}(\mathrm{OEt})_{3}\right\}_{4}$ & $7.59 \pm 1.08$ & $(0.98 \pm 0.14) \times 10^{-4}$ & STM-I $(s)$ & 67 \\
\hline & & $24.1 \pm 4.5$ & $(3.12 \pm 0.58) \times 10^{-4}$ & STM-BJ & 67 \\
\hline 23 & trans $-\mathrm{Pt}\left(\mathrm{C} \equiv \mathrm{CC}_{6} \mathrm{H}_{2}\left(\mathrm{OC}_{6} \mathrm{H}_{13}\right)_{2} \mathrm{C} \equiv \mathrm{Cpy}\right)_{2}\left(\mathrm{PPh}_{3}\right)_{2}$ & 0.76 & $9.8 \times 10^{-6}$ & STM-I $(s)$ & 68 \\
\hline 24 & trans $-\mathrm{Ru}\left(\mathrm{C} \equiv \mathrm{CC}_{6} \mathrm{H}_{2}\left(\mathrm{OC}_{6} \mathrm{H}_{13}\right)_{2} \mathrm{C} \equiv \mathrm{Cpy}\right)_{2}(\mathrm{dppe})_{2}$ & 0.35 & $4.5 \times 10^{-6}$ & STM-I $(s)$ & 68 \\
\hline 25 & trans $-\mathrm{Pt}\left(\mathrm{C} \equiv \mathrm{CC}_{6} \mathrm{H}_{2}\left(\mathrm{OC}_{6} \mathrm{H}_{13}\right)_{2} \mathrm{C} \equiv \mathrm{CC}_{6} \mathrm{H}_{4} \mathrm{SMe}\right)_{2}\left(\mathrm{PPh}_{3}\right)_{2}$ & 1.4 & $1.8 \times 10^{-5}$ & STM-I $(s)$ & 68 \\
\hline 26 & trans $-\mathrm{Ru}\left(\mathrm{C} \equiv \mathrm{CC}_{6} \mathrm{H}_{2}\left(\mathrm{OC}_{6} \mathrm{H}_{13}\right)_{2} \mathrm{C} \equiv \mathrm{CC}_{6} \mathrm{H}_{4} \mathrm{SMe}\right)_{2}(\text { dppe })_{2}$ & 1.4 & $1.8 \times 10^{-5}$ & STM-I $(s)$ & 68 \\
\hline 27 & py $\left\{\mathrm{C} \equiv \mathrm{CC}_{6} \mathrm{H}_{2}\left(\mathrm{OC}_{6} \mathrm{H}_{13}\right)_{2}\right\}_{2} \mathrm{C} \equiv \mathrm{Cpy}$ & 0.24 & $3.1 \times 10^{-6}$ & MCBJ & 42 \\
\hline 28 & py $\left\{\mathrm{C} \equiv \mathrm{CC}_{6} \mathrm{H}_{2}\left(\mathrm{OC}_{6} \mathrm{H}_{13}\right)_{2}\right\}_{3} \mathrm{C} \equiv \mathrm{Cpy}$ & 0.015 & $2.0 \times 10^{-7}$ & MCBJ & 42 \\
\hline \multirow[t]{2}{*}{$30 \mathrm{~b}$} & $\operatorname{SeCN}\left\{\mathrm{Fe}(\text { depe })_{2}\right\}(\mu-\mathrm{C} \equiv \mathrm{CC} \equiv \mathrm{C})\left\{\mathrm{Fe}(\text { depe })_{2}\right\} N C S e$ & 0.013 & $1.7 \times 10^{-7}$ & $\mathrm{MCBJ}^{\mathrm{c}}$ & 69 \\
\hline & & 0.61 & $7.9 \times 10^{-6}$ & $\mathrm{MCBJ}^{\mathrm{d}}$ & 69 \\
\hline \multirow[t]{2}{*}{$30 c$} & $\operatorname{SCN}\left\{\mathrm{Fe}(\text { depe })_{2}\right\}(\mu-\mathrm{C} \equiv \mathrm{CC} \equiv \mathrm{C})\left\{\mathrm{Fe}(\text { depe })_{2}\right\} \mathrm{NCS}$ & 0.093 & $1.2 \times 10^{-6}$ & $\mathrm{MCBJ}^{\mathrm{c}}$ & 69 \\
\hline & & 29 & $3.8 \times 10^{-4}$ & $\mathrm{MCBJ}^{\mathrm{d}}$ & 69 \\
\hline \multirow[t]{2}{*}{$30 d$} & $\mid \mathrm{C} \equiv \mathrm{C}\left\{\mathrm{Fe}(\text { depe })_{2}\right\}(\mu-\mathrm{C} \equiv \mathrm{CC} \equiv \mathrm{C})\left\{\mathrm{Fe}(\text { depe })_{2}\right\} \mathrm{C} \equiv \mathrm{C} \mid$ & 110 & $1.5 \times 10^{-3}$ & $\mathrm{MCBJ}^{\mathrm{c}}$ & 69 \\
\hline & & 1000 & $1.3 \times 10^{-2}$ & $\mathrm{MCBJ}^{\mathrm{d}}$ & 69 \\
\hline \multirow[t]{2}{*}{$30 \mathrm{e}$} & $\mid \mathrm{C} \equiv \mathrm{CC} \equiv \mathrm{C}\left\{\mathrm{Fe}(\text { depe })_{2}\right\}(\mu-\mathrm{C} \equiv \mathrm{CC} \equiv \mathrm{C})\left\{\mathrm{Fe}(\text { depe })_{2}\right\} \mathrm{C} \equiv \mathrm{CC} \equiv \mathrm{C} \mid$ & 85 & $1.1 \times 10^{-3}$ & $\mathrm{MCBJ}^{\mathrm{c}}$ & 69 \\
\hline & & 690 & $8.9 \times 10^{-3}$ & $\mathrm{MCBJ}^{\mathrm{d}}$ & 69 \\
\hline
\end{tabular}

${ }^{\mathrm{a}} 4 \mathrm{~V}$ bias. ${ }^{\mathrm{b}} \mathrm{PPh}_{3}$ contact ('short circuit'). ${ }^{\mathrm{c}} 0.2 \mathrm{~V}$ bias. ${ }^{\mathrm{d}} 1 \mathrm{~V}$ bias.

Beyond expectations: molecular conductance studies of platinum bis(acetylide) complexes

The HOMOs of square planar, $\mathrm{d}^{8}$ bis(acetylide) complexes of platinum offer similar nodal properties to those of the octahedral $\mathrm{d}^{6}$ ruthenium complexes described above, albeit at lower energy and with lower metal contribution. ${ }^{70}$

Comparisons of the electrical properties of platinum compounds trans- 
$\operatorname{Pt}(\mathrm{C} \equiv \mathrm{CR})_{2}(\mathrm{~L})_{2}(\mathrm{~L}=$ tri(alkyl $)$ or tri(aryl) phosphine with those of analogous ruthenium complexes trans $-\mathrm{Ru}(\mathrm{C} \equiv \mathrm{CR})_{2}(\mathrm{PP})_{2}$ are therefore useful in exploring structure-property relationships in metal-complex based molecular junctions.

In early work, the platinum complex trans- $\mathrm{Pt}\left(\mathrm{C} \equiv \mathrm{CC}_{6} \mathrm{H}_{4} \mathrm{SAc}\right)_{2}\left(\mathrm{PPh}_{3}\right)_{2} \quad$ (7a) (Figure 6) was investigated in a single-molecule, mechanically controlled break junction. The protecting acyl groups are removed in situ, allowing the resulting thiolates to bind to the gold electrodes. ${ }^{65}$ Under vacuum and at high bias $(4-5$ V) where Fowler-Nordheim tunnelling or field emission will likely be the dominant mechanism for through molecule conductance, the molecular resistance of 7a was determined to be $5-50 \mathrm{G} \Omega$, which equates to a molecular conductance of $2.6 \times 10^{-6}-2.6 \times 10^{-7} G_{0}$, some three orders of magnitude higher (resistance) or lower (conductance) than related oligo(arylene ethynylene) compounds. This result has lead to the perception that the low contribution from $\mathrm{Pt} d$-orbitals to the HOMO structure of complexes such as $7 \mathbf{a}$ results in broken through-molecule conjugation, and hence platinum complexes of this type might be regarded as molecular insulators rather than wires.

However, under lower bias $(100 \mathrm{mV})$ where conductance may be regarded as taking place by tunnelling through a barrier, the conductance values of a range of similar complexes trans $-\mathrm{Pt}\left(\mathrm{C} \equiv \mathrm{CC}_{6} \mathrm{H}_{4} \mathrm{SAc}\right)_{2}(\mathrm{~L})_{2}\left(\mathrm{~L}=\mathrm{PPh}_{3}\right.$ (7a), $\mathrm{PCy}_{3}(\mathbf{7 b}$, $\mathrm{Cy}=$ cyclohexyl), $\mathrm{P}(\mathrm{OEt})_{3}(7 \mathrm{c})$ ) (Figure 6) in multi-molecule cross-wire tunnel junctions were found to be essentially independent of the nature of the ancillary ligand, and some $2-3$ fold higher in conductance than the reference oligo(phenylene ethynylene) (OPE) compound 1,4-(4- $\left.\mathrm{AcSC}_{6} \mathrm{H}_{4} \mathrm{C} \equiv \mathrm{C}\right)_{2} \mathrm{C}_{6} \mathrm{H}_{4}$ (1) ${ }^{71}$ The higher conductance of the metal complexes $7 \mathbf{a}-\mathbf{c}$ is consistent with their shorter length (ca. $1.6 \AA$ shorter than 1) if one assumes that $\beta$ is in the same range as OPE compounds $\left(0.36-0.58 \AA^{-1}\right)$, despite the low Pt $d$-orbital contribution to the HOMO. However, the uncertainty over the number of molecules within the cross-wire junction precludes an accurate assessment of the single-molecule conductance. 

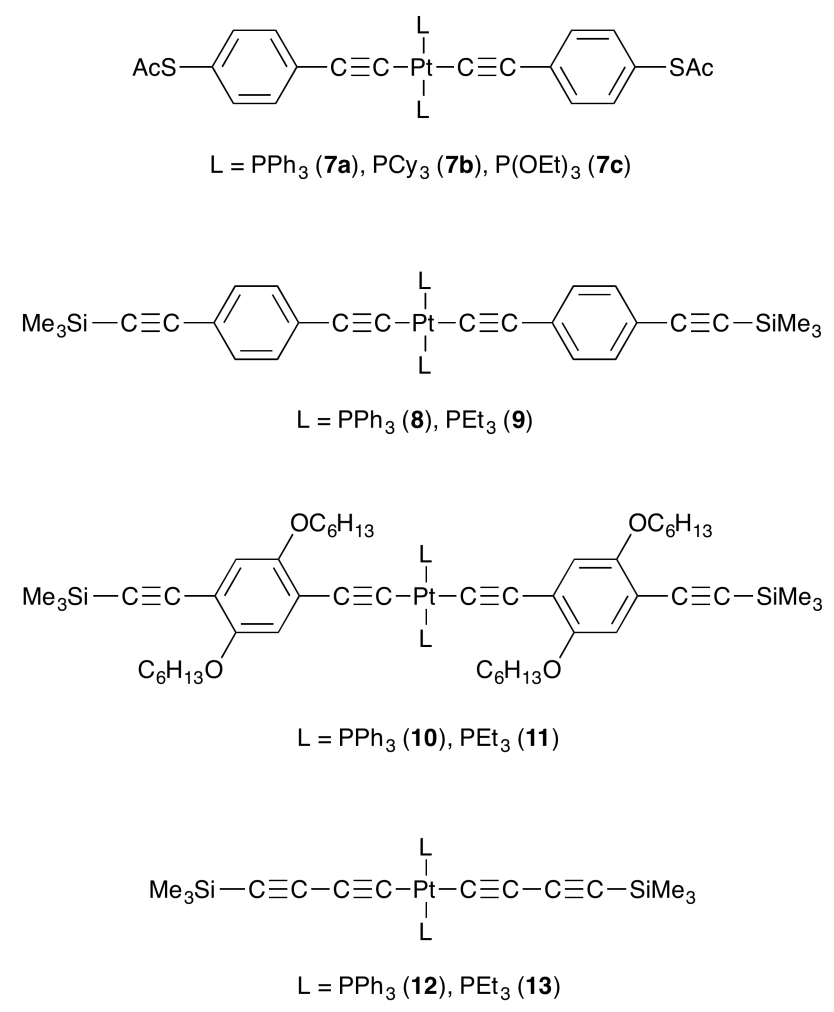

$\mathrm{Me}_{3} \mathrm{Si}-\mathrm{C} \equiv \mathrm{C}-\mathrm{C} \equiv \mathrm{C}-\mathrm{C} \equiv \mathrm{C}-\mathrm{C} \equiv \mathrm{C}-\mathrm{SiMe}_{3}$

14

$\mathrm{Me}_{3} \mathrm{Si}-\mathrm{C} \equiv \mathrm{C}-\mathrm{C} \equiv \mathrm{C}-\mathrm{C} \equiv \mathrm{C}-\mathrm{C} \equiv \mathrm{C}-\mathrm{C} \equiv \mathrm{C}-\mathrm{SiMe}_{3}$

15

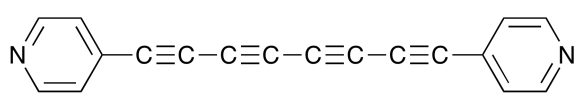

16

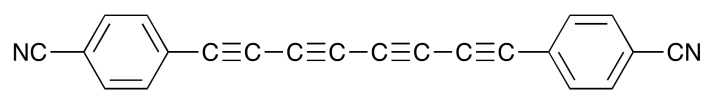

17

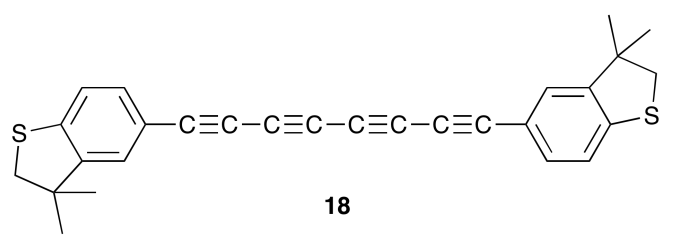

Figure 6. The complexes and compounds $7-18$

Further investigations of platinum square-planar $d^{8}$ bis(acetylide) complexes bearing different anchor groups have confirmed the generalisation of the wirelike properties of these systems. ${ }^{66}$ A series of Pt-complexes trans$\mathrm{Pt}\left(\mathrm{C} \equiv \mathrm{CC}_{6} \mathrm{H}_{4} \mathrm{C} \equiv \mathrm{CSiMe}_{3}\right)_{2} \mathrm{~L}_{2} \quad\left(\mathrm{~L}=\mathrm{PPh}_{3} \quad\right.$ (8), $\quad \mathrm{PEt}_{3} \quad$ (9)) and trans$\mathrm{Pt}\left(\mathrm{C} \equiv \mathrm{C}\left\{\mathrm{C}_{6} \mathrm{H}_{2}\left(\mathrm{OC}_{6} \mathrm{H}_{13}\right)_{2}\right\} \mathrm{C} \equiv \mathrm{CSiMe}_{3}\right)_{2} \mathrm{~L}_{2}\left(\mathrm{~L}=\mathrm{PPh}_{3}(\mathbf{1 0}), \mathrm{PEt}_{3}\right.$ (11)) (Figure 6) 
with comparable Si...Si separation (ca. $2.4 \mathrm{~nm}$ ) investigated by the STM-I(s) method gave conductance values of the order of $(3 \pm 1) \times 10^{-5} G_{0}$, independent of the nature of the substitution of the 1,4-phenylene group $\left(\mathrm{C}_{6} \mathrm{H}_{4}\right.$ or $\left.\mathrm{C}_{6} \mathrm{H}_{2}\left(\mathrm{OC}_{6} \mathrm{H}_{13}\right)_{2}\right)$ or the supporting ligands, L (Table 1). A series of DFT nonequilibrium Green's function (NEGF) calculations suggest that the tail of the LUMO resonances from these compounds overlap with the electrode Fermi levels at low bias, giving rise to a LUMO-based conductance channel, consistent with other results from trimethylsilylethynyl contacted molecules. ${ }^{25}$, 72

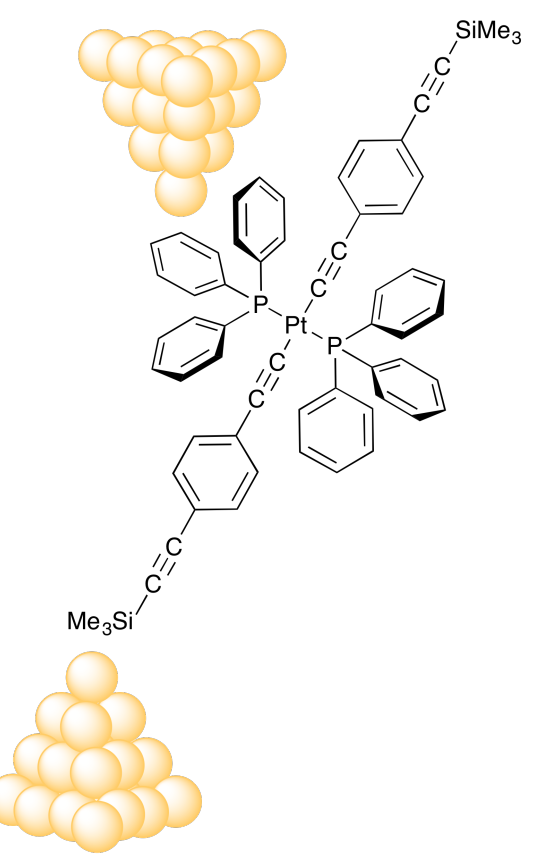

Figure 7. A molecular 'short circuit' from contact to ancillary, $\pi$-electron rich ancillary ligands in a molecular junction.

However, a word of caution is necessary in considering these data, as 'shortcircuits' formed by contacts to one of the $\mathrm{PPh}_{3}$ ligands have been identified in junctions formed from $\mathbf{8}$ and the closely related bis(buta-1,3-diynyl) complex trans $-\mathrm{Pt}\left(\mathrm{C} \equiv \mathrm{CC} \equiv \mathrm{CSiMe}_{3}\right)_{2}\left(\mathrm{PPh}_{3}\right)_{2} \quad$ (12) (Figure 7). ${ }^{66}$ Similar contacts via the dppe ligand are also likely responsible for the additional features evident in the conductance histograms of trans $-\mathrm{Ru}(\mathrm{C} \equiv \mathrm{CR})_{2}(\mathrm{dppe})_{2}$ complexes (vide infra). Although the hexyloxy $\left(\mathrm{OC}_{6} \mathrm{H}_{13}\right)$ groups in $\mathbf{1 0}$ appear to inhibit these adventitious contacts and 'insulate' the wire-like portion of the molecule, it 
may be prudent to avoid bulky, aryl phosphine and other ' $\pi$-rich' ancillary ligands as general design principle for metal complexes as molecular electronic components, and especially in cases where the system under study features a relatively weak electrode contact.

The tri(ethyl)phosphine-supported bis(buta-1,3-diynyl) complex trans$\mathrm{Pt}\left(\mathrm{C} \equiv \mathrm{CC} \equiv \mathrm{CSiMe}_{3}\right)_{2}\left(\mathrm{PEt}_{3}\right)_{2}(\mathbf{1 3})$ gave a higher conductance $\left((4.9 \pm 1.6) \times 10^{-5}\right.$ $G_{0}$ ), than 9, 10 and 11, as would be expected from the shorter Si...Si distance. However, quite remarkably, the conductance of $\mathbf{1 3}$ is also higher than that of the organic oligoynes $\mathrm{Me}_{3} \mathrm{Si}(\mathrm{C} \equiv \mathrm{C})_{4} \mathrm{SiMe}_{3}$ (14) and $\mathrm{Me}_{3} \mathrm{Si}(\mathrm{C} \equiv \mathrm{C})_{5} \mathrm{SiMe}_{3}$ (15) bearing the same trimethylsilylethynyl contacting group (Figure 6). ${ }^{25}$ This has been attributed to the lower-lying LUMO of the metal complexes, and the greater overlap of the tail of the LUMO resonance with the electrode Fermi levels that results, even at low bias. Although the significant influence of solvent on the conductance of oligoynes makes direct comparisons of data less certain, a further survey of the available conductance data from tetraynes contacted by pyridine $\left(\mathrm{py}(\mathrm{C} \equiv \mathrm{C})_{4} \mathrm{py}, \quad 16\right)$ or benzonitrile $\left(\mathrm{NCC}_{6} \mathrm{H}_{4}(\mathrm{C} \equiv \mathrm{C})_{4} \mathrm{C}_{6} \mathrm{H}_{4} \mathrm{CN}, 17\right)$ indicates that insertion of the $\mathrm{Pt}\left(\mathrm{PEt}_{3}\right)_{2}$ moiety within the polyyne backbone, as in $\mathbf{1 3}$, has no detrimental effect on the conductance vs molecular length characteristics. ${ }^{36,45}$ Only in the case of $\mathrm{BT}(\mathrm{C} \equiv \mathrm{C})_{4} \mathrm{BT} \quad$ (18) bearing the very strongly contacting dihydrobenzo $[b]$ thiophene (BT) anchor (Figure 6) does the conductance of a similar length oligoyne surpass that of $\mathbf{1 3}$, which can be explained due the increased molecule-electrode coupling.

\section{A tale of the tails: comparative molecular conductance studies of ruthenium and platinum bis(acetylide) complexes}

Consideration of the data in Table 1 reveals the surprising efficacy of the platinum-based molecular wires; for example the STM-I(s) determined conductance of $9\left((3.2 \pm 1.3) \times 10^{-5} G_{0}\right), \mathbf{1 0}\left((3.1 \pm 0.9) \times 10^{-5} G_{0}\right)$ and $11((3.2$ $\left.\pm 0.8) \times 10^{-5} G_{0}\right)$ compare well with both the trimethylsilylethynyl $\left(\mathrm{C} \equiv \mathrm{CSiMe}_{3}\right)$ 
contacted ruthenium complex trans $-\mathrm{Ru}\left(\mathrm{C} \equiv \mathrm{CC}_{6} \mathrm{H}_{4} \mathrm{C} \equiv \mathrm{CSiMe}_{3}\right)_{2}(\mathrm{dppe})_{2}$ (5: (5.10 $\left.\pm 0.99) \times 10^{-5} G_{0}\right)$ and the OPE-type molecule $1,4-\left(4-\mathrm{Me}_{3} \mathrm{SiC} \mathrm{CC}_{6} \mathrm{H}_{4}\right)_{2} \mathrm{C}_{6} \mathrm{H}_{4}(6$ : $\left.(2.75 \pm 0.56) \times 10^{-5} G_{0}\right)$ of comparable length. Despite the significant structural changes, the similarities in the conductance behaviour of 5, 6, 9, 10 and 11, which differ by less than a factor of 2 , demand closer examination of closely structurally related $\mathrm{Ru}-$ and Pt-bis(acetylide) complexes.

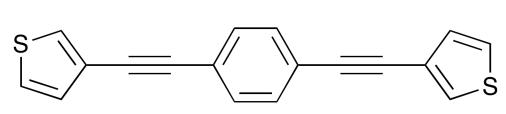

19

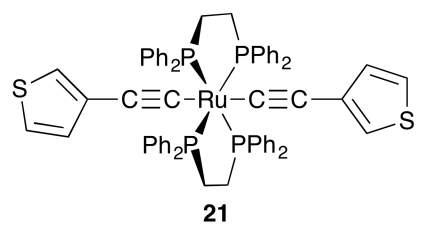

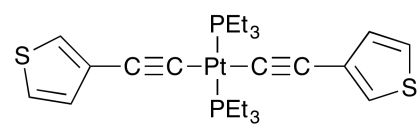

20

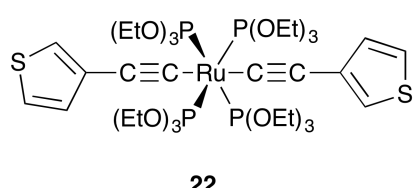

Figure 8. The compound 19 and complexes 20 - 22

The compounds $1,4-\left(3-\mathrm{SC}_{4} \mathrm{H}_{3} \mathrm{C} \equiv \mathrm{C}\right)_{2} \mathrm{C}_{6} \mathrm{H}_{4}(\mathbf{1 9})$, trans $-\mathrm{Pt}\left(\mathrm{C} \equiv \mathrm{C}-3-\mathrm{C}_{4} \mathrm{H}_{3} \mathrm{~S}\right)_{2}\left(\mathrm{PEt}_{3}\right)_{2}$ (20), trans $-\mathrm{Ru}\left(\mathrm{C} \equiv \mathrm{C}-3-\mathrm{C}_{4} \mathrm{H}_{3} \mathrm{~S}\right)_{2}(\mathrm{dppe})_{2} \quad$ (21) and trans $-\mathrm{Ru}(\mathrm{C} \equiv \mathrm{C}-3-$ $\left.\mathrm{C}_{4} \mathrm{H}_{3} \mathrm{~S}\right)_{2}\left\{\mathrm{P}(\mathrm{OEt})_{3}\right\}_{4}(\mathbf{2 2})$, in which the 3-thienyl moiety serves as the surface contacting group (Figure 8), have been examined using both STM-BJ and STM-I(s) methods. ${ }^{67}$ The rougher electrode surfaces generated by the STM-BJ method gave higher conductance groups than the STM-I(s) method which avoids contact between the STM tip and the substrate, but in each remarkably consistent sets of conductance data were obtained. The low conductance groups $\left(G_{\mathrm{L}}\right)$ obtained from STM- $I(s)$ of the platinum complex $20\left(G_{\mathrm{L}}=(0.77 \pm 0.14) \times\right.$ $\left.10^{-4} G_{0}\right)$ and ruthenium complexes $21\left((1.03 \pm 0.28) \times 10^{-4} G_{0}\right)$ and $22((0.98 \pm$ $0.14) \times 10^{-4} G_{0}$ ) were surprisingly similar, and approximately 2 -fold higher than those of the organic compound $19\left(G_{\mathrm{L}}=(0.42 \pm 0.10) \times 10^{-4} \mathrm{G}_{0}\right)$. The higher conductance data from STM-BJ $\left(G_{\mathrm{H}}\right)$ displayed even less variation $\left(G_{\mathrm{H}}: 19\right.$ $(2.83 \pm 0.65) \times 10^{-4} G_{0} ; 20(2.70 \pm 0.66) \times 10^{-4} G_{0} ; 21(3.18 \pm 1.04) \times 10^{-4} G_{0} ;$ $\left.22(3.12 \pm 0.58) \times 10^{-4} G_{0}\right)$. Additional features to the conductance peaks in the dppe complex 21 can be attributed to adventitious tip contacts to these aryl ancillary ligands. The narrower conductance peaks and absence of higher conductance side features in the conductance histograms of the alkyl phosphine 
and phosphite supported complexes $\mathbf{2 0}$ and $\mathbf{2 2}$ are consistent with this hypothesis.

The relatively high molecular conductance of the platinum complex $\mathbf{2 0}$ is consistent with the general observations noted above for $7 \mathbf{a}, \mathbf{7 b}$ and $\mathbf{7 c}$. Interestingly, basic DFT calculations indicated that at least for some of the lowlying structural minima, the HOMO contains an appreciable metal $(\mathrm{Pt})$ contribution (19\%), which is not dissimilar to the $\mathrm{Ru}$ contribution to the HOMOs of 21 (33\%) and 22 (24\%). ${ }^{67}$ Further computational analysis of the complete molecular junctions indicated that the zero-bias Fermi level lies close to the centre of the HOMO-LUMO gap in each case, albeit shifted slightly towards the tail of the HOMO resonances. Thus, despite differences in the energies of the HOMO and LUMO in each complex and the organic reference compound, transport through the middle of the HOMO-LUMO gap is similar for these (weakly coupled) thienyl-contacted molecules. The lower variation in conductance for the thienyl contacted species in comparison to the ca. 40-fold difference observed for the thiolate-contacted OPE compound $\mathbf{1}$ and ruthenium complex 2 clearly illustrates the importance of the molecule-electrode coupling, and hence the nature of the anchor group, to molecular conductance. 


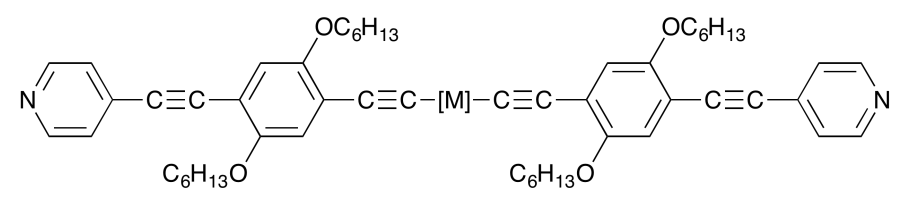

$[\mathrm{M}]=\mathrm{Pt}\left(\mathrm{PPh}_{3}\right)_{2}(\mathbf{2 3}), \mathrm{Ru}(\mathrm{dppe})_{2}(\mathbf{2 4})$

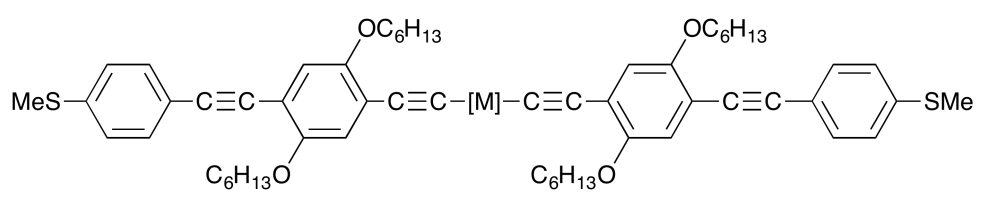

$[\mathrm{M}]=\mathrm{Pt}\left(\mathrm{PPh}_{3}\right)_{2}(25), \mathrm{Ru}(\mathrm{dppe})_{2}(\mathbf{2 6})$
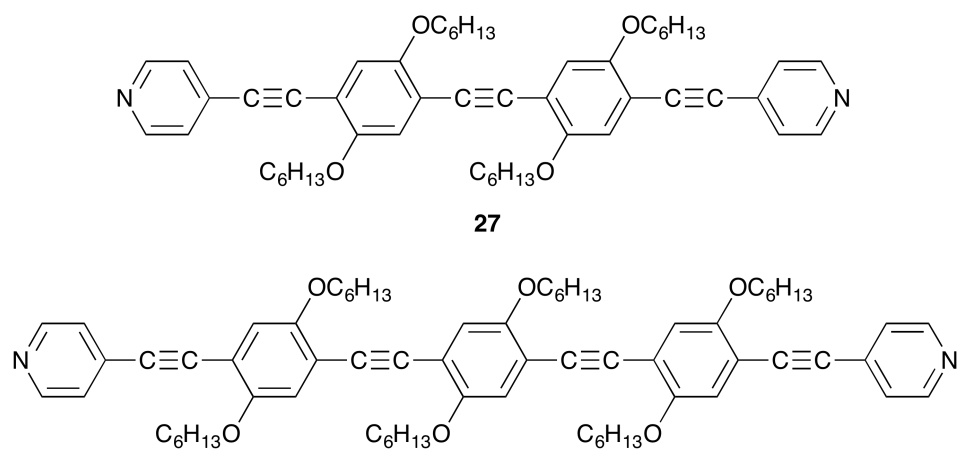

28

Figure 9. The complexes $23-28$

Further comparison of the platinum and ruthenium complexes trans$\mathrm{M}\left\{\mathrm{C} \equiv \mathrm{CC}_{6} \mathrm{H}_{2}\left(\mathrm{OC}_{6} \mathrm{H}_{13}\right)_{2} \mathrm{C} \equiv \mathrm{Cpy}\right\}_{2}\left(\mathrm{~L}_{\mathrm{n}}\right)\left[\mathrm{M}\left(\mathrm{L}_{\mathrm{n}}\right)=\mathrm{Pt}\left(\mathrm{PPh}_{3}\right)_{2}(\mathbf{2 3}), \mathrm{Ru}(\mathrm{dppe})_{2}\right.$ (24)] and trans- $\mathrm{M}\left\{\mathrm{C} \equiv \mathrm{CC}_{6} \mathrm{H}_{2}\left(\mathrm{OC}_{6} \mathrm{H}_{13}\right)_{2} \mathrm{C} \equiv \mathrm{CC}_{6} \mathrm{H}_{4} \mathrm{SMe}\right\}_{2}\left(\mathrm{~L}_{\mathrm{n}}\right)\left[\mathrm{M}\left(\mathrm{L}_{\mathrm{n}}\right)=\mathrm{Pt}\left(\mathrm{PPh}_{3}\right)_{2}(\mathbf{2 5})\right.$, $\mathrm{Ru}(\mathrm{dppe})_{2}$ (26)] (Figure 9) give additional insight into the underlying structureproperty relationships that influence molecular conductance in these systems. ${ }^{68}$ In these longer molecular wires, the platinum complexes display single molecule conductance values from STM- $I(s)$ measurements that are essentially indistinguishable from those of the comparable ruthenium examples. The SMe contacted compounds $\mathbf{2 5}$ and $\mathbf{2 6}$ give conductance values an order of magnitude higher than those of the pyridine derivatives $\mathbf{2 3}$ and $\mathbf{2 4}$, which can be attributed on the basis of DFT-calculations to the stronger Au-S bond and the consequent higher coupling and resulting broadening of the LUMO resonances. In addition, the absolute conductance values of these metal complexes are rather high for molecules of ca. $3 \mathrm{~nm}$ in length, with organic oligo(arylene ethynylene)s of similar length bearing 4-pyridyl contacts py $\left\{\mathrm{C} \equiv \mathrm{CC}_{6} \mathrm{H}_{2}\left(\mathrm{OC}_{6} \mathrm{H}_{13}\right)_{2}\right\}_{2} \mathrm{C} \equiv \mathrm{Cpy}$ (27) and py $\left\{\mathrm{C} \equiv \mathrm{CC}_{6} \mathrm{H}_{2}\left(\mathrm{OC}_{6} \mathrm{H}_{13}\right)_{2}\right\}_{3} \mathrm{C} \equiv \mathrm{Cpy}$ (28) (Figure 9) giving conductance values 
from single-molecule MCBJ measurements (in 4:1 1,3,5-trimethylbenzene : THF) $1-2$ orders of magnitude lower (Table 1$){ }^{42}$

Clearly, the insertion of a metal within the generic oligoyne or OPE type backbone generally improves the molecular conductance over an equivalent distance, and platinum complexes trans $-\mathrm{Pt}(\mathrm{C} \equiv \mathrm{CR})_{2}\left(\mathrm{~L}_{\mathrm{n}}\right)$ are not significantly worse molecular wires than similarly structured ruthenium complexes trans$\mathrm{Ru}(\mathrm{C} \equiv \mathrm{CR})_{2}\left(\mathrm{~L}_{\mathrm{n}}\right)$. This is generally found to be due to the smaller HOMOLUMO gaps and resulting increased overlap of the tails of the HOMO or LUMO resonance, broadened by the molecular coupling with the electrode, and the electrode Fermi level.

\section{1, 2, 3... Molecular conductance studies of oligomeric bis(acetylide) complexes.}

Despite the excellent conductance characteristics and low decay parameters associated with wire-like metal complexes trans-M $(\mathrm{C} \equiv \mathrm{CR})_{2}\left(\mathrm{~L}_{\mathrm{n}}\right)$, surprisingly few oligomeric complexes of the trans-bis(acetylide) type have been explored within molecular junctions. Rigaut and Frisbie have used a short series of bis(acetylide) $\mathrm{Ru}(\text { dppe })_{2}$ oligomers bearing different electrode-containing groups $\left(\mathrm{X}=-\mathrm{NC}, \quad-\mathrm{CH}_{2} \mathrm{SAc},-\mathrm{O}\left(\mathrm{CH}_{2}\right)_{6} \mathrm{SAc}\right) \quad(\mathbf{2 9 a}-\mathbf{c}(\mathbf{X})$, Figure 10). Measurements from conducting probe AFM (CP-AFM) and cross-wire junctions have been used to estimate contact group sensitive decay constants, $\beta$, in SAMs of these complexes of $0.9 \mathrm{~nm}^{-1}$ (isocyanide contacts, $\mathrm{X}=-\mathrm{NC}$ ), 1.0 $\mathrm{nm}^{-1}\left(\mathrm{X}=-\mathrm{CH}_{2} \mathrm{~S}(\mathrm{Ac})\right)$ and $1.6 \mathrm{~nm}^{-1}\left(\mathrm{X}=-\mathrm{OC}_{6} \mathrm{H}_{12} \mathrm{~S}(\mathrm{Ac})\right) .{ }^{73,74}$ These values are impressively low, and compare well with some of the very best organic compounds studied to date, including OPEs $\left(\beta\right.$ ca. $\left.2.0-3.4 \mathrm{~nm}^{-1}\right)$, oligoynes $(\beta$ ca. $\left.0.1^{25}-3.2 \mathrm{~nm}^{-1}\right)^{36}$ and porphyrin oligomers $\left(\beta\right.$ ca. $\left.0.4 \mathrm{~nm}^{-1}\right){ }^{51,52}$ The observation of a series of peaks in $d \mathrm{I} / d \mathrm{~V}$ plots that correlate with the electrochemically established oxidation processes of these complexes strongly hints at the potential for redox gating of conductance in such systems, whilst temperature dependent measurements support a transition from direct tunnelling to thermally activated hopping in the longer molecules. 


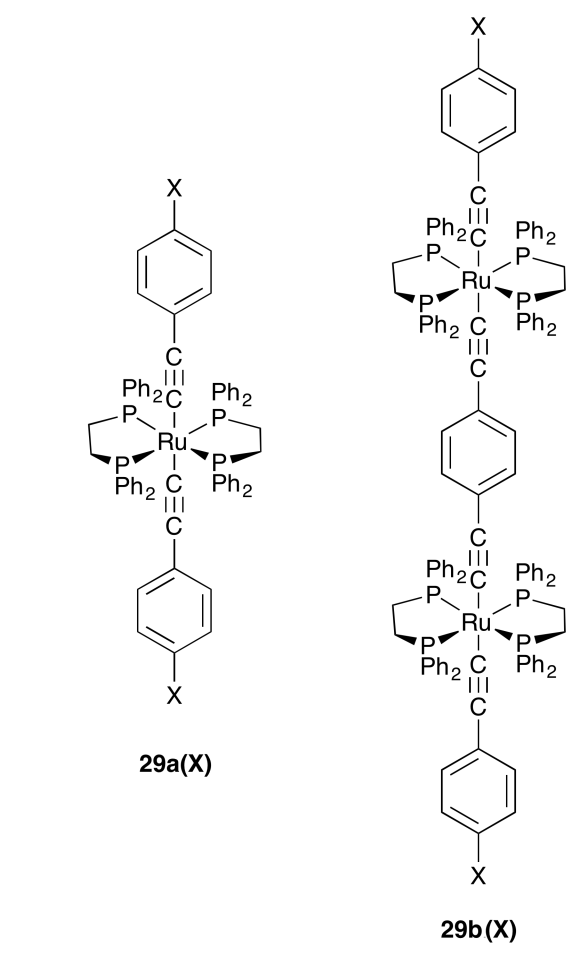

$X=-\mathrm{NC}, \mathrm{CH}_{2} \mathrm{SAC},-\mathrm{O}\left(\mathrm{CH}_{2}\right)_{6} \mathrm{SAC}$

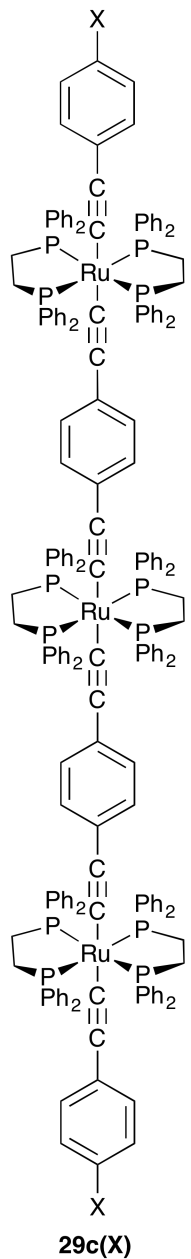

$29 c(X)$

Figure 10. Families of oligomeric trans-bis(acetylide) ruthenium complexes used in the exploration of length dependence of charge transport in molecular junctions.

Berke and colleagues have used MCBJ to explore metal complex wires featuring two $\mathrm{Fe}(\text { depe })_{2}$ moieties in the backbone, $\left[\mathrm{X}-\mathrm{Fe}(\text { depe })_{2}\right\}(\mu$ $\left.\mathrm{C} \equiv \mathrm{CC} \equiv \mathrm{C})\left\{\mathrm{Fe}(\text { depe })_{2}\right\}-\mathrm{X}\right] \quad(\mathbf{3 0}) \quad[\mathrm{X}=-\mathrm{CN}$ (a), -NCSe (b), -NCS (c), $\left.\mathrm{C} \equiv \mathrm{C}\left(\mathrm{SnMe}_{3}\right)(\mathbf{d}),-\mathrm{C} \equiv \mathrm{CC} \equiv \mathrm{C}\left(\mathrm{SnMe}_{3}\right)(\mathbf{e})\right]$ (Figure 11). The $\mathrm{X}$ group serves as the electrode contacting group, except in the case of $30 d$ and 30 e where extrusion of the trimethyltin moiety results in direct $\equiv \mathrm{C}-\mathrm{Au}$ contacts. ${ }^{69}$ Whilst the cyano $(\mathrm{X}=\mathrm{CN})$ contacted compound 30 a gave less distinct molecular conductance signatures, the other compounds gave stable junctions suitable for the measurement of conductance histograms and $I-V$ curves, and allowing exploration at room $(300 \mathrm{~K})$ and low $(30-100 \mathrm{~K})$ temperature. The currentvoltage $(I-V)$ and differential conductance-voltage $(G-V)$ curves of the more 
weakly coupled bis(iron) complexes displayed broad features and individual curves gave evidence of hysteretic conductance switching. For the - $\mathrm{CN}$, -NCS and -NCSe contacted compounds, in the low bias range $( \pm 0.25 \mathrm{~V})$, the available experimental and computational evidence indicates that transmission takes place through the tails of the resonances arising from the HOMO and HOMO1 , which are delocalised over the molecular backbone through $\pi$-d overlaps. At higher bias $( \pm 1 \mathrm{~V})$ (although lower than the threshold for field emission mechanisms to become dominant) the conductance increases by 2 orders of magnitude as the HOMOs begin to come into resonance.

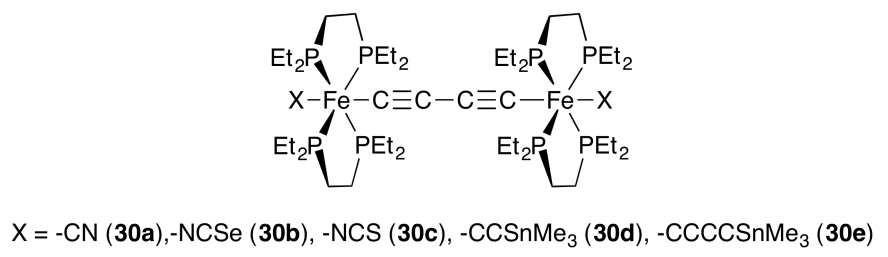

Figure 11. The complexes 30a - e

Room temperature measurements at relatively low $(0.2 \mathrm{~V})$ bias revealed exceptionally high conductance values and lower spread of values (estimated from the widths of the conductance peaks in the histograms) associated with the carbon-contacted derivatives (Table 1). At higher bias (1 V), each of these bis(iron) complexes exhibits even higher conductance, resulting in truly exceptional molecular conductance values of the order of $10^{-2} G_{0}$ for the carbon-contacted species. Computational analysis further supports the notion that the remarkably high conductance values of the carbon-contacted compounds results from the strong carbon-metal covalent bonds, and hence the molecule-electrode coupling, that further broadens the molecular levels leading to broader peaks in the transmission functions. The increased hybridisation of the molecular orbitals with the metal electrodes also shifts those molecular levels closer to the Fermi level, lowering the tunnel barrier heights and again evincing the increased conductance that results from bringing the HOMO and HOMO-1 closer to resonance with the Fermi levels. ${ }^{69}$

\section{Electrical behaviour beyond wire-like conductance}


The rich redox chemistry of the bimetallic bis(acetylide) complexes, which encompasses significant redox non-innocent character of the acetylide ligands, ${ }^{57,70,75}$ gives further opportunities to engineer switchable or modulated electrical response in molecular junctions. In an early demonstration, Rigaut, Kushmerick, Frisbie and colleagues observed peaks in the low-temperature (5 K) conductance-voltage traces from crossed-wire junctions formed from the trimetallic complex 29c(NC) (Figure 10). ${ }^{74}$ The similarity of the conductancevoltage peaks to the electrochemical processes observed by cyclic voltammetry for $29 \mathbf{c}(\mathrm{NC})$ in solution suggests that these features arise from discrete redox / electronic states that can be accessed within the bias window swept during the $I-V$ measurement.

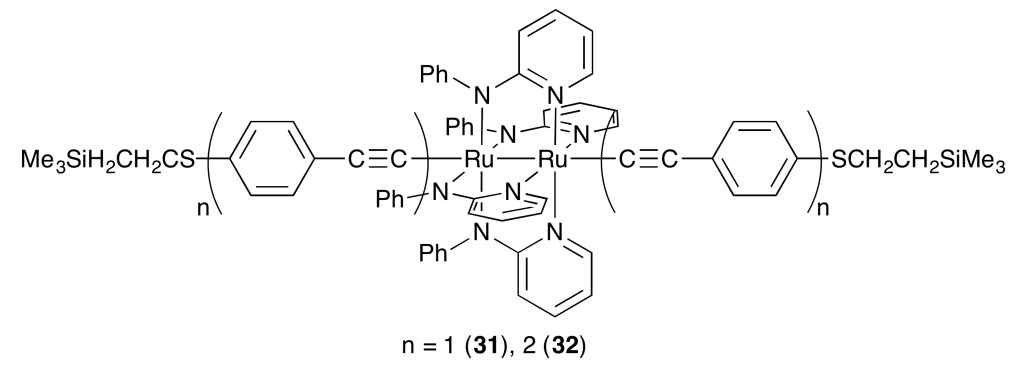

Figure 12. The bimetallic bis(acetylide) complexes 31 and 32

The bimetallic bis(acetylide) complexes trans $-\left[\mathrm{Ru}_{2}(\mu\right.$ -

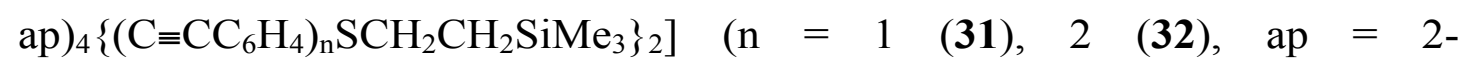
anilinopyrdinate) (Figure 12) also offer readily accessible redox states, and facile, fluoride induced deprotection of the thiol contacting groups allowed molecular junctions to be formed within electromigration-induced nanogap electrodes. ${ }^{76}$ At low bias, conductance of these multi-molecule junctions has been determined to be $(6.2 \pm 1) \times 10^{-4} G_{0}(\mathbf{3 1})$ and $(7.1 \pm 0.6) \times 10^{-5} G_{0}(\mathbf{3 2})$. Whilst the single molecule conductance cannot be accurately extracted from these data due to the uncertainty over the number of molecules within the nanogap (estimated to be $<20$ ), the decrease in conductance with molecular length $(21 \AA$ (31); $35 \AA$ (32)) is consistent with expectation. Over a larger bias range, during the sweep to $\pm \mathrm{V}$ the $I-V$ curves display a plateau (slow scan rate $0.02 \mathrm{~V} / \mathrm{s}$ ) or peak (fast scan rate $2 \mathrm{~V} / \mathrm{s}$ ) at potentials that correspond to the 
redox processes (oxidation or reduction) observed by cyclic voltammetry in solution. These higher conductance features are absent in the return sweep from $\pm \mathrm{V}$ to $0 \mathrm{~V}$. The hysteretic behaviour has been attributed to the combined effects of charging the molecule during the bias sweep which shifts the molecular level near the HOMO or LUMO nearer the Fermi level (resulting in a peak or increase in current/conductance) and, assuming the molecule remains charged, Coulomb blockade would result in a lower conductance trace during the reverse sweep. ${ }^{76}$

The observation of hysteresis in the conductance behaviour of redox-active organometallic complexes has prompted further consideration of the potential for electric-field induced conductance switching in such systems. ${ }^{77}$ Such 'two electrode' conductance switches would allow simpler construction than the more common three electrode designs such as back-gated MCBJ devices or electrochemically and electrolytically gated STM-based junctions. This phenomenon is gathering increasing interest through studies of the currentvoltage $(I-V)$ and derived conductance-voltage $(\mathrm{d} I / \mathrm{dV}-\mathrm{V})$ traces of molecular junctions which allow a greater bias window to be explored, and creating opportunities to access molecular resonances and molecular redox processes within the junction. ${ }^{9}$

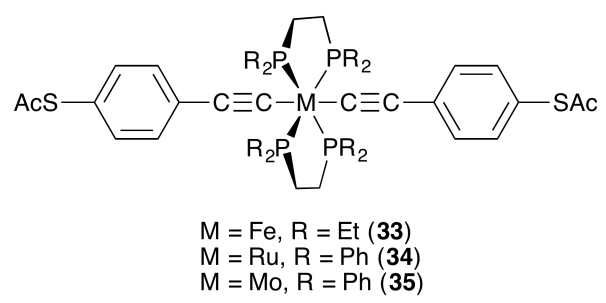

Figure 13 The complexes 33 - 35

The 18-electron $\left(\mathrm{d}^{6}\right)$ compounds trans- $\mathrm{Fe}\left(\mathrm{C} \equiv \mathrm{CC}_{6} \mathrm{H}_{4} \mathrm{SAc}\right)_{2}(\mathrm{depe})_{2}$ (33), trans$\mathrm{Ru}\left(\mathrm{C} \equiv \mathrm{CC}_{6} \mathrm{H}_{4} \mathrm{SAc}\right)_{2}(\mathrm{dppe})_{2} \quad(\mathbf{3 4}) \quad$ and 16-electron $\quad\left(\mathrm{d}^{4}\right)$ trans$\mathrm{Mo}\left(\mathrm{C} \equiv \mathrm{CC}_{6} \mathrm{H}_{4} \mathrm{SAc}\right)_{2}(\mathrm{dppe})_{2}$ (35) (Figure 13) have been explored within MCBJ structures (in which they are contacted as the thiolate) at $50 \mathrm{~K}$, with bias rapidly swept over ca. $\pm 1.1 \mathrm{~V} \cdot{ }^{77}$ All three compounds $\mathbf{3 3}-\mathbf{3 5}$ display $I-V$ curves with a small hysteresis over a narrow voltage range, with high-to-low current ratios of 
$1.5-20$. This behaviour has been attributed to the interplay between tunnelling through the molecule and a slower hopping mechanism that arises when charge becomes trapped on a less strongly coupled MO (i.e. a molecular redox process), when structural and internal electronic relaxation rates are comparable with the bias sweeping rate. In the case of the $d^{6} \mathrm{Fe}$ and $\mathrm{Ru}$ complexes, this hopping state was attributed to the HOMO-1, whilst for the $d^{4} \mathrm{Mo}^{\mathrm{II}}$ compound 35 the high-lying, metal localised $d_{\mathrm{xy}}$ orbital was found important.

Consistent with the proposed redox contribution to the overall electrical response, the switching was found to be bias sweep rate dependent, and the general features of the hysteresis could be satisfactorily modelled by fitting the probability of tunnelling vs hopping charge transfer and the bias sweeping rate within a simple two-MO tight-binding model. Interestingly, at maximum junction extension, the $d^{4} \mathrm{Mo}^{\mathrm{II}}$ compound $\mathbf{3 5}$ also displayed a second type of hysteretic conductance behaviour. Whilst these 'type II' curves were some 100 times lower current, the high-to-low current ratios exceeded 1000:1. Whilst the weaker metal-electrode coupling in these fully extended junctions accounts for the overall lower currents, the larger hysteresis effect can be attributed to the lower thermodynamic driving force for the hopping mechanism arising in part from the higher reorganisation energy associated with the redox processes in the fully extended junction. ${ }^{77}$

The capacity to change conductance through molecular redox (charging) processes within a junction leads to concepts of more sophisticated electronic components such as transistors and in memory applications based on redoxactive molecules. With this in mind, the remarkable success of Ren and his team in developing flash-style memories from redox-active bimetallic alkynyl complexes $\mathrm{Ru}_{2}(\mathrm{C} \equiv \mathrm{CR})(\text { ap) })_{4}$ (ap = 2-anilinopyridinate) is a sign-post to further opportunities in the field. ${ }^{78}$ Indeed, the exploration and exploitation of molecular redox processes to engineer electrical response within a molecular junction beyond non-resonant tunnelling based conductance looms as the next area for significant exploration in the field of molecular electronics, and is an area in which metal complexes are likely ideally placed to find application. 
The logical next step: exploiting redox and photochemical functions of the ligands

The 'modular' synthetic pathways employed for the synthesis of metal bis(acetylide) complexes allows considerable scope for the introduction of acetylide ligands bearing chemical, photochemical and additional redox functionality which can be used in combination with the redox character associated with the metal bis(acetylide) moiety. ${ }^{56}$

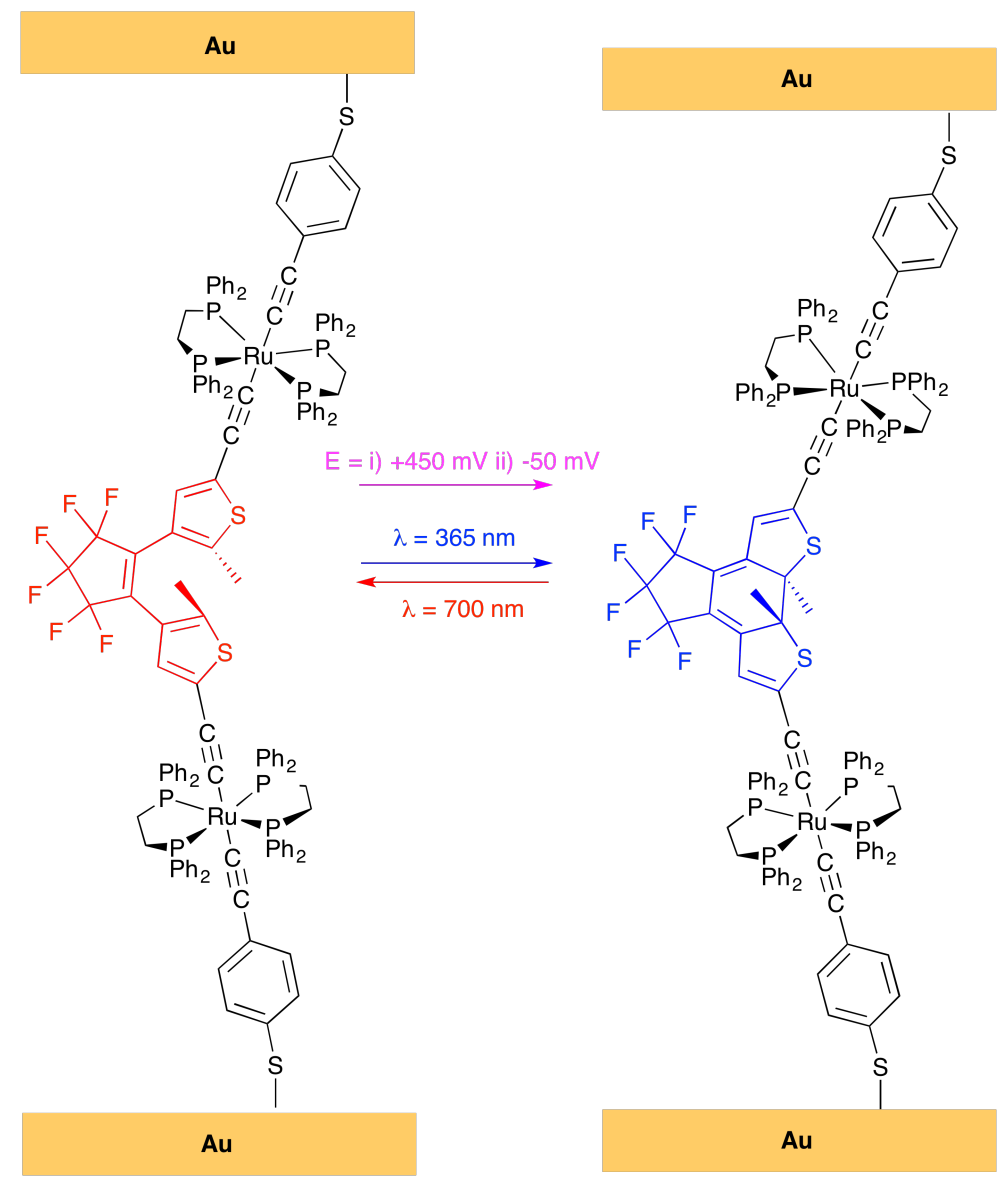

Figure 14 The photochemical switching of complex 36 between 'open' lower conductance (left) and 'closed' higher conductance (right) isomers. The ringclosing isomerisation can also be achieved through a cycle of electrochemical oxidation ( $E=+450 \mathrm{mV} v \mathrm{Ag} / \mathrm{AgCl})$, radical ring closing, and reduction (-50 $m V)$.

This strategy has been used to design photo-modulable molecular components in which a dithienylethene (DTE) moiety is used to bridge two $\mathrm{Ru}(\mathrm{dppe})_{2}$ based 
bis(acetylide) fragments (36), which are in turn functionalised by thioacetate moieties to provide for contact to gold electrodes (Figure 14). ${ }^{79}$ The DTE framework undergoes a facile photo-induced rearrangement from a crossconjugated 'open' state to a linearly conjugated 'closed' state under the influence of UV light. The process can be reversed under visible light, giving a bistable system capable of reversible switching between these isomeric states. When assembled within a wire-based nanogap, the resulting multi-molecular junction formed from the initially closed form was able to undergo smooth switching between high and low conductance states. The electrical response could be readily linked to the underlying molecular and electronic structures of the two isomeric forms, with the higher lying HOMO of the closed form giving rise to a peak in the transmission curve nearer the electrode Fermi level under conditions of both zero and $0.6 \mathrm{~V}$ bias. The metal fragment and thiolate linker combination also appear to play an important role in limiting quenching of the photochemical response from the electrode surface, pointing to further opportunities for the use of metal fragments in multi-functional molecular component designs. $^{79}$ 


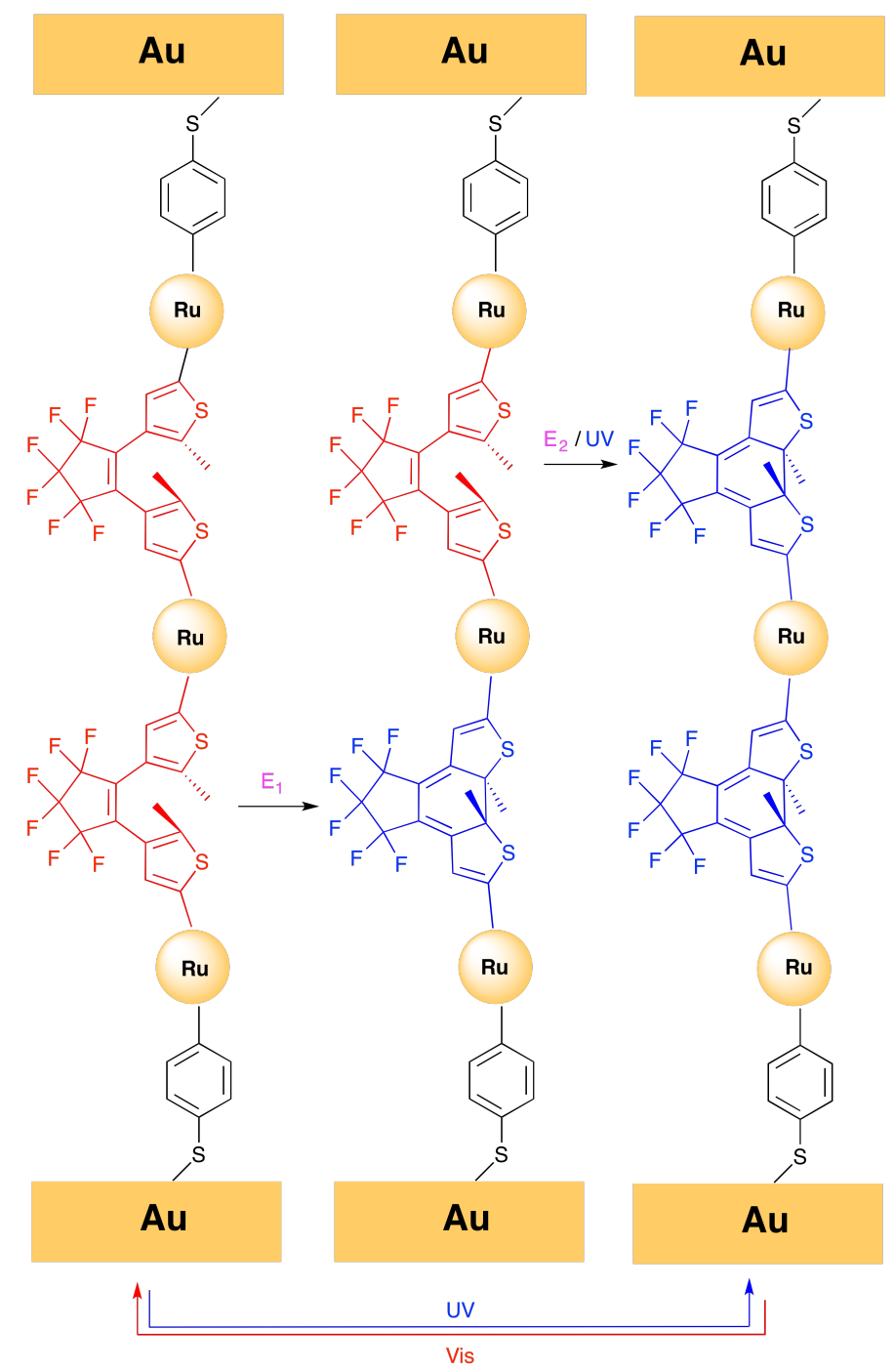

Figure 15 The orthogonal modulation of the open and closed forms of 37 . (Ru $=$ trans-Ru(dppe $)_{2} ; U V=365 \mathrm{~nm} ; \mathrm{Vis}=700 \mathrm{~nm} ; E_{1}, E_{2}=+450 \mathrm{mV} /-50 \mathrm{mV}$ vs $\mathrm{Ag} / \mathrm{AgCl}$ ).

The combination of the redox-activity of the metal bis(acetylide) fragment with the photoactivity of the DTE cores makes compounds such as $\mathbf{3 6}$ and the trimetallic homologue 37 powerful platforms through which to demonstrate orthogonally modulated electrical response in a molecular junction. ${ }^{80}$ The 'open' and 'closed' forms of $\mathbf{3 6}$ and $\mathbf{3 7}$ undergo electrochemical oxidation at rather different potentials, as would be expected from the differing HOMO compositions and energies that underpin the different conductance properties outlined above for 36, allowing for selective photochemical and electrochemical addressing. As illustrated in Figure 14, the open (low conductance / high resistance) form of $\mathbf{3 6}$ can be isomerised to the closed form 
through application of an external electrochemical potential in an electrochemically-gated nano-gap junction, or by UV (365 nm) irradiation. The process can be reversed through a suitable reduction potential or visible (700 $\mathrm{nm})$ light. The electrochemically and optically gated junction therefore functions as a simple OR gate. The additional DTE moiety in $\mathbf{3 7}$ gave additional functionality, and whilst complete independent addressing of the DTE fragments could not be achieved, sufficient control through photochemical and electrochemical processes was realised to give an elementary, resettable AND-OR gate (Figure 15). ${ }^{80}$

\section{Summary and outlook}

Metal complexes trans- $\mathrm{M}(\mathrm{C} \equiv \mathrm{CR})_{2}\left(\mathrm{~L}_{\mathrm{n}}\right)$ offer a range of chemical features that are useful in the study of the electrical characteristics of molecular junctions and explorations of through molecule conductance. The molecular conductance of trans $-\mathrm{M}(\mathrm{C} \equiv \mathrm{CR})_{2}\left(\mathrm{~L}_{\mathrm{n}}\right)$ systems is generally greater than that of typical conjugated organic compounds with the same anchor groups, due to the typically smaller HOMO-LUMO gaps or closer alignment of frontier orbitals with the Fermi energies of (gold) electrodes at low bias. However, conductance under low bias is still dominated by transmission through the tails of HOMO (or LUMO in the case of extended alkynyl ligands) resonances with the Fermi level falling more or less in the middle of the large HOMO-LUMO gap of these stable molecules. It is clear that the anchor groups, which dictate the moleculeelectrode coupling and broaden the molecular resonances, play an important role in the overall electrical characteristics of the junction and in many cases the influence of the anchor group on the junction conductance exceeds that of the nature of the metal. To this end, the identification of strongly coupled electrode-carbon contacts and the development of routes to these systems promise to be valuable entry points to highly conductive wire-like molecular systems.

The HOMO-LUMO gap plays a role in determining both the overlap of the molecular resonances with the Fermi level and also in the conductance vs 
length characteristics (i.e. the decay constant, $\beta$ ). Looking to the design of future systems, stable open shell (radical) compounds that offer characteristically small HOMO-LUMO (or HOSO-LUSO) gaps, as evinced by the typically colourful nature of these species, will likely become attractive targets for further investigation.

The robust redox processes associated with many metal complexes trans$\mathrm{M}(\mathrm{C} \equiv \mathrm{CR})_{2}\left(\mathrm{~L}_{\mathrm{n}}\right)$ and demonstration of redox-gated switching of conductance hints at the scope for these compounds to find use in the development of transistor-like response in three-electrode junctions. Similarly the ability to access and trap different charge states in two-electrode systems during higher bias sweeps has given early evidence of bi-stable switching and opportunities for development of novel memory systems.

The chemical space for exploration is enlarged further when functional ligands are introduced to the generic trans $-\mathrm{M}(\mathrm{C} \equiv \mathrm{CR})_{2}\left(\mathrm{~L}_{\mathrm{n}}\right)$ system. In many molecular component designs presented to date, the alkynyl ligands have been designed to provide a delocalised $\pi$-electron channel for the effective transmission of charge and wire-like response in the resulting junctions. However, the possibility to use chemical functionality within the ligand backbone opens many exciting opportunities for introducing electrical (redox), optical (photochemical) and sensing (chemical) function within the junction.

The electrical response of molecular junctions is clearly highly dependent on the nature of the molecule-electrode interface and the resulting electronic coupling term, which are in turn strongly dependent on the nature of the anchor group. Whilst the broadening of molecular states that accompanies strong metal-molecule coupling is beneficial for conductance, the hybridisation of molecule and electrode electronic characteristics may suppress the optoelectronic response of individual molecular fragments. Thus, through consideration of the role of metal-electrode coupling and careful design it has proven possible to not only retain functionality in modular compounds but also to address and exploit these characteristics in junctions that exhibit memory and 
logic function. The design and synthesis of molecules with multiple 'integrated' electronic functions and assembly within single molecular circuits is therefore now emerging as an achievable objective, supported by ever increasing understanding of the structure-property relationships that underpin the electrical characteristics of molecular junctions. The chemical, structural, optical and redox diversity offered by trans-M(C $\equiv \mathrm{CR})_{2}\left(\mathrm{~L}_{\mathrm{n}}\right)$ ensures that these compounds will continue to play an important role in the development of the next generation of molecular electronic components, devices and circuits.

\section{Conflicts of interest}

There are no conflicts to declare.

\section{Acknowledgements}

The authors wish to acknowledge the discussions with many of the authors of the works cited and discussed that form the basis of this article. We particularly acknowledge the support and encouragement from Professors Richard Nichols, Simon Higgins and Colin Lambert, who have been instrumental in our own activities in this field.

\section{Notes and references}

1. S. Marques-Gonzalez and P. J. Low, Aust J Chem, 2016, 69, 244-253.

2. D. Xiang, X. L. Wang, C. C. Jia, T. Lee and X. F. Guo, Chem Rev, 2016, 116, 4318-4440.

3. R. J. Nichols and S. J. Higgins, Annu Rev Anal Chem, 2015, 8, 389-417.

4. Y. Komoto, S. Fujii, M. Iwane and M. Kiguchi, J Mater Chem C, 2016, 4, $8842-8858$.

5. C. J. Lambert, H. Sadeghi and Q. H. Al-Galiby, C. R. Phys, 2016, 17, 10841095.

6. V. M. Garcia-Suarez, C. J. Lambert, D. Z. Manrique and T. Wandlowski, Nanotechnology, 2014, 25.

7. A. Vezzoli, I. Grace, C. Brooke, K. Wang, C. J. Lambert, B. Q. Xu, R. J. Nichols and S. J. Higgins, Nanoscale, 2015, 7, 18949-18955.

8. J. Y. Liu, X. T. Zhao, Q. Al-Galiby, X. Y. Huang, J. T. Zheng, R. H. Li, C. C. Huang, Y. Yang, J. Shi, D. Z. Manrique, C. J. Lambert, M. R. Bryce and W. J. Hong, Angew Chem Int Ed, 2017, 56, 13061-13065.

9. R. J. Nichols and S. J. Higgins, Acc Chem Res, 2016, 49, 2640-2648. 
10. J. Park, A. N. Pasupathy, J. I. Goldsmith, C. Chang, Y. Yaish, J. R. Petta, M. Rinkoski, J. P. Sethna, H. D. Abruna, P. L. McEuen and D. C. Ralph, Nature, 2002, 417, 722-725.

11. W. J. Liang, M. P. Shores, M. Bockrath, J. R. Long and H. Park, Nature, 2002, 417, 725-729.

12. C. Herrmann, G. C. Solomon and M. A. Ratner, J Am Chem Soc, 2010, 132, 3682-3684.

13. R. Liu, S. H. Ke, W. Yang and H. U. Baranger, J Chem Phys, 2007, 127, 144107.

14. C. J. Lambert, Chem Soc Rev, 2015, 44, 875-888.

15. M. Kiguchi and S. Kaneko, Phys Chem Chem Phys, 2013, 15, 2253-2267.

16. T. A. Su, M. Neupane, M. L. Steigerwald, L. Venkataraman and C. Nuckolls, Nat Rev Mater, 2016, 1, 1-15.

17. F. Chen, J. Hihath, Z. F. Huang, X. L. Li and N. J. Tao, Annu Rev Phys Chem, 2007, 58, 535-564.

18. C. Shu, C. Z. Li, H. X. He, A. Bogozi, J. S. Bunch and N. J. Tao, Phys Rev Lett, 2000, 84, 5196-5199.

19. C. Z. Li, H. X. He and N. J. Tao, Appl Phys Lett, 2000, 77, 3995-3997.

20. C. Z. Li and N. J. Tao, Appl Phys Lett, 1998, 72, 894-896.

21. S. Martin, I. Grace, M. R. Bryce, C. S. Wang, R. Jitchati, A. S. Batsanov, S. J. Higgins, C. J. Lambert and R. J. Nichols, J Am Chem Soc, 2010, 132, 91579164.

22. J. Ulrich, D. Esrail, W. Pontius, L. Venkataraman, D. Millar and L. H. Doerrer, J Phys Chem B, 2006, 110, 2462-2466.

23. W. Haiss, S. Martin, E. Leary, H. van Zalinge, S. J. Higgins, L. Bouffier and R. J. Nichols, J Phys Chem C, 2009, 113, 5823-5833.

24. E. Leary, H. Hobenreich, S. J. Higgins, H. van Zalinge, W. Haiss, R. J. Nichols, C. M. Finch, I. Grace, C. J. Lambert, R. McGrath and J. Smerdon, Phys Rev Lett, 2009, 102, 086801.

25. D. C. Milan, O. A. Al-Owaedi, M. C. Oerthel, S. Marques-Gonzalez, R. J. Brooke, M. R. Bryce, P. Cea, J. Ferrer, S. J. Higgins, C. J. Lambert, P. J. Low, D. Z. Manrique, S. Martin, R. J. Nichols, W. Schwarzacher and V. M. GarciaSuarez, J Phys Chem C, 2016, 120, 15666-15674.

26. M. Kiguchi, O. Tal, S. Wohlthat, F. Pauly, M. Krieger, D. Djukic, J. C. Cuevas and J. M. van Ruitenbeek, Phys Rev Lett, 2008, 101, 046801.

27. M. Iwane, S. Fujii, T. Nishino and M. Kiguchi, J Phys Chem C, 2016, 120, 8936-8940.

28. S. Kaneko, C. Motta, G. P. Brivio and M. Kiguchi, Nanotechnology, 2013, 24, 315201.

29. Z. L. Cheng, R. Skouta, H. Vazquez, J. R. Widawsky, S. Schneebeli, W. Chen, M. S. Hybertsen, R. Breslow and L. Venkataraman, Nat Nanotechnol, 2011, 6, 353-357.

30. W. B. Chen, J. R. Widawsky, H. Vazquez, S. T. Schneebeli, M. S. Hybertsen, R. Breslow and L. Venkataraman, J Am Chem Soc, 2011, 133, 17160-17163.

31. M. Kiguchi and K. Murakoshi, J Phys Chem C, 2008, 112, 8140-8143.

32. M. Kiguchi, Appl Phys Lett, 2009, 95, 0733301.

33. F. Chen, X. L. Li, J. Hihath, Z. F. Huang and N. J. Tao, J Am Chem Soc, 2006, 128, 15874-15881.

34. L. Venkataraman, J. E. Klare, I. W. Tam, C. Nuckolls, M. S. Hybertsen and M. L. Steigerwald, Nano Lett, 2006, 6, 458-462. 
35. X. L. Li, J. He, J. Hihath, B. Q. Xu, S. M. Lindsay and N. J. Tao, J Am Chem Soc, 2006, 128, 2135-2141.

36. P. Moreno-Garcia, M. Gulcur, D. Z. Manrique, T. Pope, W. J. Hong, V. Kaliginedi, C. C. Huang, A. S. Batsanov, M. R. Bryce, C. Lambert and T. Wandlowski, J Am Chem Soc, 2013, 135, 12228-12240.

37. W. J. Hong, D. Z. Manrique, P. Moreno-Garcia, M. Gulcur, A. Mishchenko, C. J. Lambert, M. R. Bryce and T. Wandlowski, J Am Chem Soc, 2012, 134, 2292-2304.

38. E. J. Dell, B. Capozzi, K. H. DuBay, T. C. Berkelbach, J. R. Moreno, D. R. Reichman, L. Venkataraman and L. M. Campos, J Am Chem Soc, 2013, 135, 11724-11727.

39. Y. P. Zang, A. Pinkard, Z. F. Liu, J. B. Neaton, M. L. Steigerwald, X. Roy and L. Venkataraman, J Am Chem Soc, 2017, 139, 14845-14848.

40. S. Schneebeli, M. Kamenetska, F. Foss, H. Vazquez, R. Skouta, M. Hybertsen, L. Venkataraman and R. Breslow, Org Lett, 2010, 12, 4114-4117.

41. M. T. Gonzalez, X. T. Zhao, D. Z. Manrique, D. Miguel, E. Leary, M. Gulcur, A. S. Batsanov, G. Rubio-Bollinger, C. J. Lambert, M. R. Bryce and N. Agrait, J Phys Chem C, 2014, 118, 21655-21662.

42. X. T. Zhao, C. C. Huang, M. Gulcur, A. S. Batsanov, M. Baghernejad, W. J. Hong, M. R. Bryce and T. Wandlowski, Chem Mater, 2013, 25, 4340-4347.

43. Q. Lu, K. Liu, H. M. Zhang, Z. B. Du, X. H. Wang and F. S. Wang, Acs Nano, 2009, 3, 3861-3868.

44. H. M. Osorio, S. Martin, M. C. Lopez, S. Marques-Gonzalez, S. J. Higgins, R. J. Nichols, P. J. Low and P. Cea, Beilstein J Nanotech, 2015, 6, 1145-1157.

45. C. S. Wang, A. S. Batsanov, M. R. Bryce, S. Martin, R. J. Nichols, S. J. Higgins, V. M. Garcia-Suarez and C. J. Lambert, J Am Chem Soc, 2009, 131, 15647-15654.

46. H. M. Liu, N. Wang, J. W. Zhao, Y. Guo, X. Yin, F. Y. C. Boey and H. Zhang, Chemphyschem, 2008, 9, 1416-1424.

47. H. X. Li, M. H. Garner, T. A. Su, A. Jensen, M. S. Inkpen, M. L. Steigerwald, L. Venkataraman, G. C. Solomon and C. Nuckolls, J Am Chem Soc, 2017, 139, 10212-10215.

48. V. Kaliginedi, P. Moreno-Garcia, H. Valkenier, W. J. Hong, V. M. GarciaSuarez, P. Buiter, J. L. H. Otten, J. C. Hummelen, C. J. Lambert and T. Wandlowski, J Am Chem Soc, 2012, 134, 5262-5275.

49. S. H. Choi, B. Kim and C. D. Frisbie, Science, 2008, 320, 1482-1486.

50. R. Yamada, H. Kumazawa, T. Noutoshi, S. Tanaka and H. Tada, Nano Lett, 2008, 8, 1237-1240.

51. G. Sedghi, V. M. Garcia-Suarez, L. J. Esdaile, H. L. Anderson, C. J. Lambert, S. Martin, D. Bethell, S. J. Higgins, M. Elliott, N. Bennett, J. E. Macdonald and R. J. Nichols, Nat Nanotechnol, 2011, 6, 517-523.

52. G. Sedghi, K. Sawada, L. J. Esdaile, M. Hoffmann, H. L. Anderson, D. Bethell, W. Haiss, S. J. Higgins and R. J. Nichols, J Am Chem Soc, 2008, 130, 8582-8583.

53. S. J. Higgins and R. J. Nichols, Polyhedron, 2018, 140, 25-34.

54. Y. Tanaka, M. Kiguchi and M. Akita, Chem-Eur J, 2017, 23, 4741-4749.

55. P. J. Low, Dalton Trans, 2005, 2821-2824.

56. X. Y. He, C. Lagrost, L. Norel and S. Rigaut, Polyhedron, 2018, 140, 169180. 
57. S. Marques-Gonzalez, M. Parthey, D. S. Yufit, J. A. K. Howard, M. Kaupp and P. J. Low, Organometallics, 2014, 33, 4947-4963.

58. Y. S. Park, A. C. Whalley, M. Kamenetska, M. L. Steigerwald, M. S. Hybertsen, C. Nuckolls and L. Venkataraman, J Am Chem Soc, 2007, 129, 15768-15769.

59. C. Vericat, M. E. Vela, G. Corthey, E. Pensa, E. Cortes, M. H. Fonticelli, F. Ibanez, G. E. Benitez, P. Carro and R. C. Salvarezza, RSC Adv, 2014, 4, 27730-27754.

60. E. Pensa, E. Cortes, G. Corthey, P. Carro, C. Vericat, M. H. Fonticelli, G. Benitez, A. A. Rubert and R. C. Salvarezza, Acc Chem Res, 2012, 45, 11831192.

61. H. Hakkinen, Nat Chem, 2012, 4, 443-455.

62. K. Liu, X. H. Wang and F. S. Wang, ACS Nano, 2008, 2, 2315-2323.

63. K. Sugimoto, Y. Tanaka, S. Fujii, T. Tada, M. Kiguchi and M. Akita, Chem Commun, 2016, 52, 5796-5799.

64. S. Marques-Gonzalez, D. S. Yufit, J. A. K. Howard, S. Martin, H. M. Osorio, V. M. Garcia-Suarez, R. J. Nichols, S. J. Higgins, P. Cea and P. J. Low, Dalton Trans, 2013, 42, 338-341.

65. M. Mayor, C. von Hanisch, H. B. Weber, J. Reichert and D. Beckmann, Angew Chem Int Ed, 2002, 41, 1183-+.

66. O. A. Al-Owaedi, S. Bock, D. C. Milan, M. C. Oerthel, M. S. Inkpen, D. S. Yufit, A. N. Sobolev, N. J. Long, T. Albrecht, S. J. Higgins, M. R. Bryce, R. J. Nichols, C. J. Lambert and P. J. Low, Nanoscale, 2017, 9, 9902-9912.

67. S. Bock, O. A. Al-Owaedi, S. G. Eaves, D. C. Milan, M. Lemmer, B. W. Skelton, H. M. Osorio, R. J. Nichols, S. J. Higgins, P. Cea, N. J. Long, T. Albrecht, S. Martin, C. J. Lambert and P. J. Low, Chem-Eur J, 2017, 23, 2133-2143.

68. O. A. Al-Owaedi, D. C. Milan, M. C. Oerthel, S. Bock, D. S. Yufit, J. A. K. Howard, S. J. Higgins, R. J. Nichols, C. J. Lambert, M. R. Bryce and P. J. Low, Organometallics, 2016, 35, 2944-2954.

69. F. Schwarz, G. Kastlunger, F. Lissel, H. Riel, K. Venkatesan, H. Berke, R. Stadler and E. Lortscher, Nano Lett, 2014, 14, 5932-5940.

70. M. Parthey, K. B. Vincent, M. Renz, P. A. Schauer, D. S. Yufit, J. A. K. Howard, M. Kaupp and P. J. Low, Inorg Chem, 2014, 53, 1544-1554.

71. T. L. Schull, J. G. Kushmerick, C. H. Patterson, C. George, M. H. Moore, S. K. Pollack and R. Shashidhar, J Am Chem Soc, 2003, 125, 3202-3203.

72. R. R. Ferradas, S. Marques-Gonzalez, H. M. Osorio, J. Ferrer, P. Cea, D. C. Milan, A. Vezzoli, S. J. Higgins, R. J. Nichols, P. J. Low, V. M. GarciaSuarez and S. Martin, RSC Adv, 2016, 6, 75111-75121.

73. L. Luo, A. Benameur, P. Brignou, S. H. Choi, S. Rigaut and C. D. Frisbie, $J$ Phys Chem C, 2011, 115, 19955-19961.

74. B. Kim, J. M. Beebe, C. Olivier, S. Rigaut, D. Touchard, J. G. Kushmerick, X. Y. Zhu and C. D. Frisbie, J Phys Chem C, 2007, 111, 7521-7526.

75. P. A. Schauer and P. J. Low, Eur J Inorg Chem, 2012, 390-411.

76. A. K. Mahapatro, J. W. Ying, T. Ren and D. B. Janes, Nano Lett, 2008, 8, 2131-2136.

77. F. Schwarz, G. Kastlunger, F. Lissel, C. Egler-Lucas, S. N. Semenov, K. Venkatesan, H. Berke, R. Stadler and E. Lortscher, Nat Nanotechnol, 2016, 11, 170-176. 
78. S. Pookpanratana, H. Zhu, E. G. Bittle, S. N. Natoli, T. Ren, C. A. Richter, Q. Li and C. A. Hacker, J Phys-Condens Mat, 2016, 28, 094009.

79. F. B. Meng, Y. M. Hervault, L. Norel, K. Costuas, C. Van Dyck, V. Geskin, J. Cornil, H. H. Hng, S. Rigaut and X. D. Chen, Chem Sci, 2012, 3, 3113-3118.

80. F. B. Meng, Y. M. Hervault, Q. Shao, B. H. Hu, L. Norel, S. Rigaut and X. D. Chen, Nat Commun, 2014, 5, 3023. 


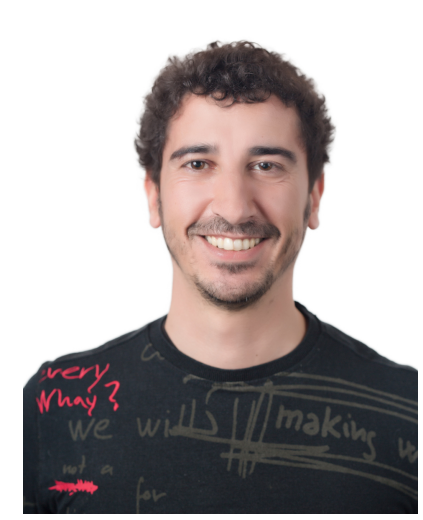

J. Nichols.

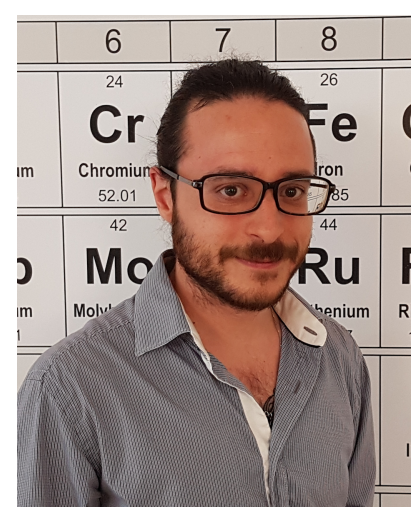

Dr. Andrea Vezzoli obtained his $\mathrm{PhD}$ in Chemistry from the University of Liverpool in 2015, supervised by Prof. Simon J. Higgins, working on the synthesis of compounds for molecular electronics studies. He has remained in Liverpool as a research associate where his work is now focused on the fabrication of hybrid metal-molecule-semiconductor devices for spintronics applications, under the supervision of Prof. Richard J. Nichols. His research interests lie in the synthetic preparation of organic and organometallic molecular wires, the fabrication of single-molecule junctions, and the development of scanning probe methods for the characterisation of self-assembled single entities.

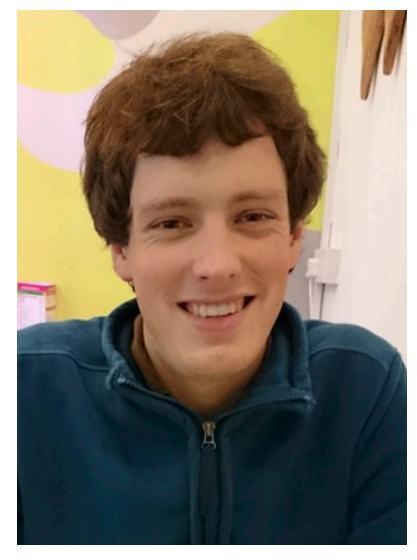

Mr Inco Planje graduated from his undergraduate Chemistry degree in the Netherlands in 2015 . He then moved to Liverpool for a $\mathrm{PhD}$ in the molecular electronics group of Prof. Richard J. Nichols and Prof. Simon J. Higgins. His research project focuses on single-molecule electronic measurements, particularly on metal complexes and wires containing novel anchoring groups. Another of his research interests involves machine-learning techniques for 


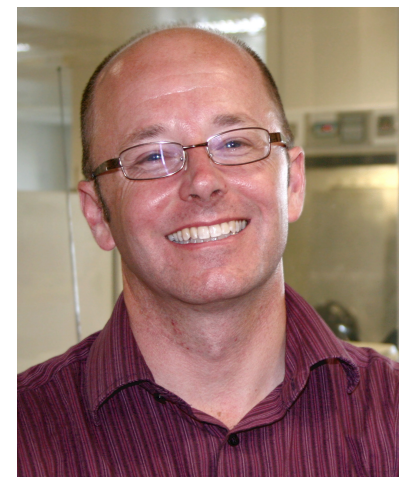

Prof Paul J. Low studied at the University of Adelaide, where he obtained his $\mathrm{PhD}$ (1997) under the supervision of Prof. Michael I. Bruce. After postdoctoral work at the Canadian National Research Council (Steacie Institute for Molecular Sciences) with Prof. Arthur J. Carty (1997 - 1999), he was appointed to a Lectureship in the Department of Chemistry at Durham University (UK), being promoted to Reader (2006) and Professor (2010). In 2013 he returned to Australia as Professor of Chemistry at the University of Western Australia. Paul's interests span synthetic and redox chemistry, with particular interests in mixed-valence systems and molecular electronics. 